\title{
A Non-interactive Range Proof with Constant Communication
}

\author{
Rafik Chaabouni ${ }^{1,2}$, Helger Lipmaa ${ }^{1}$, and Bingsheng Zhang $^{1}$ \\ 1 Institute of Computer Science, University of Tartu, Estonia \\ 2 Security and Cryptography Laboratory, EPFL, Switzerland
}

\begin{abstract}
In a range proof, the prover convinces the verifier in zeroknowledge that he has encrypted or committed to a value $a \in[0, H]$ where $H$ is a public constant. Most of the previous non-interactive range proofs have been proven secure in the random oracle model. We show that one of the few previous non-interactive range proofs in the common reference string (CRS) model, proposed by Yuen et al. in COCOON 2009 , is insecure. We then construct a secure non-interactive range proof that works in the CRS model. The new range proof can have (by different instantiations of the parameters) either very short communication (14080 bits) and verifier's computation (81 pairings), short combined CRS length and communication $\left(\log ^{1 / 2+o(1)} H\right.$ group elements), or very efficient prover's computation $(\Theta(\log H)$ exponentiations).
\end{abstract}

Keywords: NIZK, pairings, progression-free sets, range proof.

\section{Introduction}

In a range proof, the prover convinces the verifier in zero-knowledge that he has encrypted or committed to a value $a \in[0, H]$, where $H$ is a public constant. Range proofs are needed in a wide variety of cryptographic protocols, like e-voting (to show that a ballot corresponds to a valid candidate), e-auctions, anonymous credentials, e-cash, or any other protocol that needs for its correctness that the inputs are from a valid range. Given the need for range proofs in a large variety of protocols, it is not surprising that there is a large amount of research on this topic.

Most of the existing efficient range proofs fall in one of the next two categories. The first category uses a classical result of Lagrange that every non-negative integer is a sum of four squares [13721. However, in this case the underlying group has to be of unknown order which seriously limits the available cryptographic techniques. In particular, all known secure Lagrange's theorem based range proofs are based on operations in $\mathbb{Z}_{n}^{*}$ for a hard-to-factor $n$. Since to achieve 128 -bit security level, $n$ must be at least 3072 bits long, arithmetic in $\mathbb{Z}_{n}^{*}$ is relatively slow. One also has to compute the four squares of the Lagrange's theorem which is inefficient by itself. Furthermore, this means that it is not known how to instantiate such schemes with bilinear groups. (This is exemplified by the fact 
that we break the range proof of [21] where the Lagrange theorem is used in the bilinear setting with known group order.)

Due to such considerations, one usually considers the second approach. There, one uses the fact that $a \in[0, H]$, if and only if for some well chosen coefficients $G_{i}$, there exist $b_{i} \in[0, u-1]$ such that $a=\sum_{i=1}^{n} G_{i} b_{i}$. Here, $u \ll H$ and $n$ is also small. One then proves separately for every $b_{i}$ that $b_{i} \in[0, u-1]$, and uses additively homomorphic properties of the used commitment scheme to verify that $a=\sum_{i=1}^{n} G_{i} b_{i}$. The goal is to minimize the communication (which is approximately $n$ times the cost of a more basic proof that $b_{i} \in[0, u-1]$ ) of that type of range proofs.

Clearly, $a \in\left[0,2^{d}-1\right]$ iff $a=\sum_{i=1}^{d} 2^{i-1} b_{i}$ and $b_{i} \in\{0,1\}$. Then one can prove that $a \in[0, H]$ for arbitrary $H$ by showing that both $a$ and $H-a$ belong to $\left[0,2^{\left\lfloor\log _{2} H\right\rfloor+1}-1\right]$. Showing that $b_{i} \in\{0,1\}$ is straightforward, e.g., by using an AND of two $\Sigma$-protocols. This means that one has to execute two basic range proofs for $\left[0,2^{d}-1\right]$. Lipmaa, Asokan and Niemi showed in [16] that by choosing the coefficients $G_{i}$ cleverly, one obtains a simpler result that $a \in[0, H]$, for any $H>1$, iff $a=\sum_{i=1}^{\left\lfloor\log _{2} H\right\rfloor+1} G_{i} b_{i}$ and $b_{i} \in\{0,1\}$.

In [3], the authors considered the general case $u \geq 2$, following the fact that $a \in\left[0, u^{d}-1\right]$ iff $a=\sum_{i=1}^{d} u^{i} b_{i}$ and $b_{i} \in[0, u-1]$. They showed that $b_{i} \in[0, u-1]$ by letting the verifier to sign every integer in $[0, u-1]$, and then letting the prover to prove that he knows the signature on committed $b_{i}$. One can show that $a \in[0, H]$ for general $H$ by using an AND of two $\Sigma$-protocols. Nontrivially generalizing [16] (by using methods from additive combinatorics), Chaabouni, Lipmaa and shelat 4 showed that there exist (efficiently computable) coefficients $G_{i}$ such that $(u-1) a \in(u-1) \cdot[0, H]$ iff $a=\sum_{i=1}^{\left\lceil\log _{u}((u-1) \cdot H+1)\right\rceil} G_{i} b_{i}$ for some $b_{i} \in[0, u-1]$. The range proof from [4 has the communication complexity of $\Theta\left(\log _{u} H+u\right)$ group elements, which obtains the minimal value $\Theta(\log H / \log \log H)$ if $u \approx \log H / \log \log H$. (See [9] for recent related work.)

Usually, it is desired that the range proof is non-interactive. For example, in the e-voting scenario, range proof is a part of the vote validity proof that is verified by various parties without any active participation of the voter. Most of the previous non-interactive range proofs first construct a $\Sigma$-protocol which is then made non-interactive in the random oracle model by using the Fiat-Shamir heuristic. While the random oracle model allows to construct efficient protocols, it is also known that there exist protocols that are secure in the random oracle models and insecure in the plain model.

Motivated by this, 52118] have proposed non-interactive range proofs without random oracles. The range proof from [5] is of mainly theoretical value. The range proof from [21] uses Lagrange's theorem, but we will demonstrate an attack on it. The range proof from [18] combines the range proof of [3] with the GrothSahai non-interactive zero-knowledge (NIZK) proofs 11] and P-signatures. The range proof from [18] is not claimed to be zero-knowledge (only NIWI, that is, non-interactive witness-indistinguishable).

We first show that the protocol from 21 is insecure. Their protocol works in a group of known order. In this case, using Lagrange's theorem to prove that a 
non-negative number is the sum of four squares fails. We can only conclude that the sum of four squares is computed modulo the group order. Hence an attacker can prove that any number is "non-negative" and completely break the protocol in [21]. See Sect. 4 for more information.

We then construct a new NIZK range proof (for an encrypted $a$ - if one needs $a$ to be committed, one can use the same cryptosystem as a perfectly binding commitment) that works in the common-reference string model. We do this by using recent NIZK arguments by Groth and Lipmaa [15]. We also use the additive combinatorics results from [4], that is, we base a range proof $a \in[0, H]$ on the fact that $(u-1) a \in(u-1) \cdot[0, H]$ iff $a=\sum_{i=1}^{n} G_{i} b_{i}$ and $b_{i} \in[0, u-1]$, where $G_{i}$ are as defined in [4. However, differently from [4, we prove that $b_{i} \in[0, u-1]$ by proving (by a recursive use of the method from [16 4]) that $b_{i}=\sum_{j=0}^{n_{v}} G_{j}^{\prime} b_{j i}^{\prime}$ with $b_{j i}^{\prime} \in[0,1]$. Here, $n_{v}:=\left\lfloor\log _{2}(u-1)\right\rfloor$.

By using the commitment scheme of [8]15] that enables to succinctly commit to a vector $\left(b_{1}, \ldots, b_{n}\right)$, and the Hadamard product argument of [815], we can do all $n_{v}+1$ small range proofs in parallel. In addition, in Sect. 5] we construct a new non-interactive argument that a knowledge-commited value is equal to a BBS-encrypted [2] value. (Due to the use of knowledge assumptions, this proof is computationally more efficient than the one constructed by using Groth-Sahai proofs [1].) The new range proof does not rely on the random oracle model or use any proofs of knowledge of signatures.

The conceptual novelty of the new range proof as compared to all previous range proofs of the "second approach" is that in all latter schemes, $a \in[0, H]$ is proven by executing in parallel $N \approx \log _{u} H$ smaller zero-knowledge proofs of type $b_{i} \in[0, u-1]$. In the new range proof, $N$ elements $b_{i}$ are arranged in an $n_{v} \times n$ matrix, where it takes only one zero-knowledge proof (the complexity of which depends on $n$ ) to prove that all elements in one row belong to the range $[0, u-1]$. By appropriately choosing the values $n_{v}$ and $n$ (and $u$ ), one can achieve different complexity trade-offs.

The complexity of the new range proof is described in Tbl. 1. Setting $u=2$ results in a constant argument length (but CRS of $\Theta\left((\log H)^{1+o(1)}\right)$ group elements). By using an efficient variation of Barreto-Naehrig curves (where the group elements are either 256 or 512 bits), the communication drops to 14080 bits. The range proof of [18] does not allow for constant communication. Moreover, if $u=2$ then the communication is even smaller than that of the known range proofs based on the Lagrange's theorem like [13]. We note that constant communication is achieved since the new range proof uses permutation arguments only for permutations that do not depend on the statement. On the other hand, setting $u=H$ results in summatory CRS and argument length of $\log ^{1 / 2+o(1)} H$, and setting $u=2^{\sqrt{\log H}}$ results in prover's computational complexity dominated by $\Theta(\log H)$ exponentiations. The previous non-interactive range proofs did not allow for such a flexibility.

One can obtain a zap (that is, a 2-message public-coin witnessindistinguishable proof) from the NIZK range proof by first letting the verifier create and send a CRS to the prover, and then letting the prover to send 
Table 1. Comparison of NIZK arguments for range proof. Here, M/E/P means the number of multiplications, exponentiations and pairings. Communication is given in group elements. Here, $n_{v}=\lfloor\log (u-1)\rfloor, n \approx \log H / \log u$ and $\varepsilon=o(1)$, and the basis of all logarithms is 2 . To fit in page margins, in this table only, we write $h=\log _{2} H$.

\begin{tabular}{l|r|r|r|r}
\hline & CRS length & Argument length & Prover comp. & Verifier comp. \\
\hline \hline 18 & $\Theta(1)$ & $\Theta(h)$ & $\Theta(h)$ & $\Theta(h)$ \\
$\underline{18}$ & $\Theta\left(\frac{h}{\log h}\right)$ & $\Theta\left(\frac{h}{\log h}\right)$ & $\Theta\left(\frac{h}{\log h}\right)$ & $\Theta\left(\frac{h}{\log h}\right)$ \\
\hline
\end{tabular}

\begin{tabular}{|c|c|c|c|c|}
\hline \multicolumn{5}{|c|}{ This paper } \\
\hline General & $n^{1+\varepsilon}$ & $5 n_{v}+40$ & $\Theta\left(n^{2} n_{v}\right) \mathrm{M}+\Theta\left(n^{1+o(1)} n_{v}\right) \mathrm{E}$ & $\left(9 n_{v}+81\right) \mathrm{P}$ \\
\hline$u=2$ & $h^{1+\varepsilon}$ & 40 & $\Theta\left(h^{2}\right) \mathrm{M}+h^{1+\varepsilon} \mathrm{E}$ & $81 \mathrm{P}$ \\
\hline$u=2^{\sqrt{h}}$ & $h^{1 / 2+\varepsilon}$ & $\approx 5 \sqrt{h}+40$ & $\Theta\left(h^{3 / 2}\right) \mathrm{M}+h^{1+\varepsilon} \mathrm{E}$ & $\approx(9 \sqrt{h}+81) \mathrm{P}$ \\
\hline$u=H$ & $\Theta(1)$ & $\approx 5 h+40$ & $\Theta(h) \mathrm{E}$ & $\approx(9 h+81) \mathrm{P}$ \\
\hline
\end{tabular}

the range proof to the verifier. This zap works in the standard model (without needing a CRS since it is generated on run) and has the total communication $\log ^{1 / 2+o(1)} H$ in the case $u=H$.

\section{Preliminaries}

Let $[L, H]=\{L, L+1, \ldots, H-1, H\}$ and $[H]=[1, H]$. Let $S_{n}$ be the set of permutations from $[n]$ to $[n]$. By $\boldsymbol{a}$, we denote the vector $\boldsymbol{a}=\left(a_{1}, \ldots, a_{n}\right)$. If $A$ is a value, then $x \leftarrow A$ means that $x$ is set to $A$. If $A$ is a set, then $x \leftarrow A$ means that $x$ is picked uniformly and randomly from $A$. If $y=h^{x}$, then $\operatorname{let} \log _{h} y:=x$. Let $\kappa$ be the security parameter. We abbreviate probabilistic polynomial-time as $\mathrm{PPT}$, and let $\operatorname{negl}(\kappa)$ be a negligible function. We say that $\Lambda=\left(\lambda_{1}, \ldots, \lambda_{n}\right) \subset \mathbb{Z}$ is an $(n, \kappa)$-nice tuple, if $0<\lambda_{1}<\cdots<\lambda_{i}<\cdots<\lambda_{n}=\operatorname{poly}(\kappa)$.

By using notation from additive combinatorics, if $\Lambda_{1}$ and $\Lambda_{2}$ are subsets of some additive group $\left(\mathbb{Z}\right.$ or $\mathbb{Z}_{p}$ within this paper $)$, then $\Lambda_{1}+\Lambda_{2}=\left\{\lambda_{1}+\lambda_{2}: \lambda_{1} \in\right.$ $\left.\Lambda_{1} \wedge \lambda_{2} \in \Lambda_{2}\right\}$ is their sum set and $\Lambda_{1}-\Lambda_{2}=\left\{\lambda_{1}-\lambda_{2}: \lambda_{1} \in \Lambda_{1} \wedge \lambda_{2} \in \Lambda_{2}\right\}$ is their difference set. If $\Lambda$ is a set, then $k \Lambda=\left\{\lambda_{1}+\cdots+\lambda_{k}: \lambda_{i} \in \Lambda\right\}$ is an iterated sumset, and $k \cdot \Lambda=\{k \lambda: \lambda \in \Lambda\}$ is a dilation of $\Lambda$. Let $2^{\wedge} \Lambda=\left\{\lambda_{1}+\lambda_{2}: \lambda_{1} \in\right.$ $\left.\Lambda \wedge \lambda_{2} \in \Lambda \wedge \lambda_{1} \neq \lambda_{2}\right\} \subseteq \Lambda+\Lambda$ denote a restricted sumset [20].

A set $\left\{\lambda_{1}, \ldots, \lambda_{n}\right\} \subset \mathbb{Z}^{+}$is progression-free, if no three of the numbers are in arithmetic progression, so that $\lambda_{i}+\lambda_{j}=2 \lambda_{k}$ only if $i=j=k$. Let $r_{3}(N)$ denote the cardinality of the largest progression-free set that belongs to $[N]$. Recently, Elkin [6] showed that $r_{3}(N)=\Omega\left(\left(N \cdot \log _{2}^{1 / 4} N\right) / 2^{2 \sqrt{2 \log _{2} N}}\right)$. It is also known that $r_{3}(N)=O\left(N(\log \log N)^{5} / \log N\right)$ [19]. Thus, the minimal $N$ such that $r_{3}(N)=n$ is $\omega(n)$, while according to Elkin, $N=n^{1+o(1)}$.

Fact 1 (Lipmaa [15]). For any fixed $n>0$, there exists $N=n^{1+o(1)}$, such that $[N]$ contains a progression-free subset $\Lambda$ of odd integers of cardinality $n$. 
Bilinear Groups. Let $\mathcal{G}_{\mathrm{bp}}\left(1^{\kappa}\right)$ be a bilinear group generator that outputs a description of a bilinear group gk $:=\left(p, \mathbb{G}_{1}, \mathbb{G}_{2}, \mathbb{G}_{T}, \hat{e}\right) \leftarrow \mathcal{G}_{\mathrm{bp}}\left(1^{\kappa}\right)$ such that $p$ is a $\kappa$-bit prime, $\mathbb{G}_{1}, \mathbb{G}_{2}$ and $\mathbb{G}_{T}$ are multiplicative cyclic groups of order $p, \hat{e}: \mathbb{G}_{1} \times$ $\mathbb{G}_{2} \rightarrow \mathbb{G}_{T}$ is a bilinear map (pairing) such that $\forall a, b \in \mathbb{Z}, t \in\{1,2\}$ and $g_{t} \in \mathbb{G}_{t}$, $\hat{e}\left(g_{1}^{a}, g_{2}^{b}\right)=\hat{e}\left(g_{1}, g_{2}\right)^{a b}$. If $g_{t}$ generates $\mathbb{G}_{t}$ for $t \in\{1,2\}$, then $\hat{e}\left(g_{1}, g_{2}\right)$ generates $\mathbb{G}_{T}$. Moreover, it is efficient to decide the membership in $\mathbb{G}_{1}, \mathbb{G}_{2}$ and $\mathbb{G}_{T}$, group operations and the pairing $\hat{e}$ are efficiently computable, generators are efficiently sampleable, and the descriptions of the groups and group elements each are $O(\kappa)$ bit long. One can implement an optimal (asymmetric) Ate pairing [12 over a subclass of Barreto-Naehrig curves [117] very efficiently. In that case, at security level of 128 -bits, an element of $\mathbb{G}_{1} / \mathbb{G}_{2} / \mathbb{G}_{T}$ can be represented in respectively $256 / 512 / 3072$ bits.

A bilinear group generator $\mathcal{G}_{\mathrm{bp}}$ is DLIN (decisional linear) secure 2] in group $\mathbb{G}_{t}$, for $t \in\{1,2\}$, if for all non-uniform PPT adversaries $\mathcal{A}$, the next probability is negligible in $\kappa$ :

$$
\left|\operatorname{Pr}\left[\begin{array}{l}
\mathrm{gk} \leftarrow \mathcal{G}_{\mathrm{bp}}\left(1^{\kappa}\right), \\
(f, h) \leftarrow\left(\mathbb{G}_{t}^{*}\right)^{2},(\sigma, \tau) \leftarrow \mathbb{Z}_{p}^{2}: \\
\mathcal{A}\left(\mathrm{gk} ; f, h, f^{\sigma}, h^{\tau}, g_{t}^{\sigma+\tau}\right)=1
\end{array}\right]-\operatorname{Pr}\left[\begin{array}{l}
\mathrm{gk} \leftarrow \mathcal{G}_{\mathrm{bp}}\left(1^{\kappa}\right), \\
(f, h) \leftarrow\left(\mathbb{G}_{t}^{*}\right)^{2},(\sigma, \tau, z) \leftarrow \mathbb{Z}_{p}^{3}: \\
\mathcal{A}\left(\mathrm{gk} ; f, h, f^{\sigma}, h^{\tau}, g_{t}^{z}\right)=1
\end{array}\right]\right|
$$

Let $\Lambda$ be an $(n, \kappa)$-nice tuple for some $n=\operatorname{poly}(\kappa)$. A bilinear group generator $\mathcal{G}_{\mathrm{bp}}$ is $\Lambda-P S D L$ secure, if for any non-uniform PPT adversary $\mathcal{A}$,

$$
\operatorname{Pr}\left[\begin{array}{l}
\mathrm{gk}:=\left(p, \mathbb{G}_{1}, \mathbb{G}_{2}, \mathbb{G}_{T}, \hat{e}\right) \leftarrow \mathcal{G}_{\mathrm{bp}}\left(1^{\kappa}\right), g_{1} \leftarrow \mathbb{G}_{1} \backslash\{1\}, \\
g_{2} \leftarrow \mathbb{G}_{2} \backslash\{1\}, x \leftarrow \mathbb{Z}_{p}: \mathcal{A}\left(\mathrm{gk} ;\left(g_{1}^{x^{s}}, g_{2}^{x^{s}}\right)_{s \in\{0\} \cup \Lambda}\right)=x
\end{array}\right]=\operatorname{negl}(\kappa) .
$$

Let $\Lambda$ be an $(n, \kappa)$-nice tuple. According to [15, any successful generic adversary for $\Lambda$-PSDL requires time $\Omega\left(\sqrt{p / \lambda_{n}}\right)$ where $p$ is the group order and $\lambda_{n}$ is the largest element of $\Lambda$.

The soundness of NIZK arguments (for example, an argument that a computationally binding commitment scheme commits to 0) seems to be an unfalsifiable assumption in general. We will use a weaker version of soundness in the case of subarguments, but in the case of the range proof, we will prove soundness. Similarly to [815], we will base the soundness of that argument on an explicit knowledge assumption.

For two algorithms $\mathcal{A}$ and $X_{\mathcal{A}}$, we write $(y ; z) \leftarrow\left(\mathcal{A} \| X_{\mathcal{A}}\right)(x)$ if $\mathcal{A}$ on input $x$ outputs $y$, and $X_{\mathcal{A}}$ on the same input (including the random tape of $\mathcal{A}$ ) outputs $z$. Let $\Lambda$ be an $(n, \kappa)$-nice tuple for some $n=\operatorname{poly}(\kappa)$. Consider $t \in\{1,2\}$. The bilinear group generator $\mathcal{G}_{\mathrm{bp}}$ is $\Lambda$-PKE secure in group $\mathbb{G}_{t}$ if for any non-uniform PPT adversary $\mathcal{A}$ there exists a non-uniform PPT extractor $X_{\mathcal{A}}$,

$$
\operatorname{Pr}\left[\begin{array}{l}
\mathrm{gk}:=\left(p, \mathbb{G}_{1}, \mathbb{G}_{2}, \mathbb{G}_{T}, \hat{e}\right) \leftarrow \mathcal{G}_{\mathrm{bp}}\left(1^{\kappa}\right), g_{t} \leftarrow \mathbb{G}_{t} \backslash\{1\}, \\
(\hat{\alpha}, x) \leftarrow \mathbb{Z}_{p}^{2}, \mathrm{crs} \leftarrow\left(\mathrm{gk} ;\left(g_{t}^{x^{s}}, g_{t}^{\hat{\alpha} x^{s}}\right)_{s \in\{0\} \cup \Lambda}\right), \\
\left(c, \hat{c} ;\left(a_{s}\right)_{s \in\{0\} \cup \Lambda} \leftarrow\left(\mathcal{A} \| X_{\mathcal{A}}\right)(\mathrm{crs}): \hat{c}=c^{\hat{\alpha}} \wedge c \neq \prod_{s \in\{0\} \cup \Lambda} g_{t}^{a_{s} x^{s}}\right.
\end{array}\right]=\operatorname{negl}(\kappa) .
$$


Groth [8] proved that the $[n]$-PKE assumption holds in the generic group model; his proof can be modified to the general case.

In the case of both the PSDL and PKE assumptions, we will define straightforward generalizations in Sect. 5.

BBS Cryptosystem. A public-key cryptosystem $\left(\mathcal{G}_{\mathrm{pkc}}, \mathcal{E} \mathrm{nc}, \mathcal{D e c}\right)$ is a triple of efficient algorithms (key generation, encryption, and decryption), where for any $(\mathrm{sk}, \mathrm{pk}) \leftarrow \mathcal{G}_{\mathrm{pkc}}\left(1^{\kappa}\right)$ and any valid $m$ and randomizer $r, \mathcal{D e c}_{\mathrm{sk}}\left(\mathcal{E}_{\mathrm{nc}} \mathrm{pk}(m ; r)\right)=$ $m$. A cryptosystem is IND-CPA secure, if for any (sk, pk) $\leftarrow \mathcal{G}_{\mathrm{pkc}}\left(1^{\kappa}\right)$ and any two messages $m_{0}$ and $m_{1}$, the distributions $\mathcal{E} \mathrm{nc}_{\mathrm{pk}}\left(m_{0} ; \cdot\right)$ and $\mathcal{E}_{\mathrm{nc}} \mathrm{pk}_{\mathrm{pk}}\left(m_{1} ; \cdot\right)$ are computationally indistinguishable. In the lifted BBS cryptosystem [2] (in group $\left.\mathbb{G}_{1}\right)$, the system parameters are equal to $\left(\mathrm{gk} ; g_{1}\right)$, where gk $\leftarrow \mathcal{G}_{\mathrm{bp}}\left(1^{\kappa}\right)$ and $g_{1} \leftarrow$ $\mathbb{G}_{1} \backslash\{1\}$. The secret key sk is $\left(\mathrm{sk}_{1}, \mathrm{sk}_{2}\right) \leftarrow\left(\mathbb{Z}_{p}^{*}\right)^{2}$, the public key pk is $(f, h) \leftarrow$ $\left(g_{1}^{1 / \mathrm{sk}_{1}}, g_{1}^{1 / \mathrm{sk}_{2}}\right)$. One encrypts $a \in \mathbb{Z}_{p}$ as $\mathcal{E} \mathrm{nc}_{\mathrm{pk}}\left(\mathrm{ck}_{1} ; a ; r_{f}, r_{h}\right) \leftarrow\left(c_{g}, c_{f}, c_{h}\right)=$ $\left(g_{1}^{r_{f}+r_{h}+a}, f^{r_{f}}, h^{r_{h}}\right)$, where $\left(r_{f}, r_{h}\right) \leftarrow \mathbb{Z}_{p}^{2}$. One decrypts $\left(c_{g}, c_{f}, c_{h}\right)$ by returning the discrete logarithm of $c_{g} /\left(c_{f}^{\text {sk }_{1}} c_{h}^{\text {sk }_{2}}\right)$. The BBS cryptosystem is IND-CPA secure under the DLIN assumption.

Commitment Schemes in the CRS Model. A (batch) commitment scheme $\left(\mathcal{G}_{\text {com }}, \mathcal{C}\right.$ om $)$ in a bilinear group consists of two PPT algorithms: a randomized CRS generation algorithm $\mathcal{G}_{\text {com }}$, and a randomized commitment algorithm $\mathcal{C}$ om. Here, $\mathcal{G}_{\text {com }}^{t}\left(1^{\kappa}, n\right), t \in\{1,2\}$, produces a $\operatorname{CRS~} \mathrm{ck}_{t}$, and $\mathcal{C o m}^{t}\left(\mathrm{ck}_{t} ; \boldsymbol{a} ; r\right)$, with $\boldsymbol{a}=\left(a_{1}, \ldots, a_{n}\right)$, outputs a commitment value $A$ in $\mathbb{G}_{t}^{b}$ for $b>1$ (in our case, $b=2$ or $b=3)$. A commitment $\mathcal{C o m}^{t}\left(\mathrm{ck}_{t} ; \boldsymbol{a} ; r\right)$ is opened by revealing $(\boldsymbol{a}, r)$.

A commitment scheme $\left(\mathcal{G}_{\text {com }}, \mathcal{C}\right.$ om $)$ is computationally binding in group $\mathbb{G}_{t}$, if for every non-uniform PPT adversary $\mathcal{A}$ and positive integer $n=\operatorname{poly}(\kappa)$,

$$
\operatorname{Pr}\left[\begin{array}{l}
\mathrm{ck}_{t} \leftarrow \mathcal{G}_{\mathrm{com}}^{t}\left(1^{\kappa}, n\right),\left(\boldsymbol{a}_{\mathbf{1}}, r_{1}, \boldsymbol{a}_{\mathbf{2}}, r_{2}\right) \leftarrow \mathcal{A}\left(\mathrm{ck}_{t}\right): \\
\left(\boldsymbol{a}_{\mathbf{1}}, r_{1}\right) \neq\left(\boldsymbol{a}_{\mathbf{2}}, r_{2}\right) \wedge \mathcal{C o m}^{t}\left(\mathrm{ck}_{t} ; \boldsymbol{a}_{\mathbf{1}} ; r_{1}\right)=\mathcal{C o m}^{t}\left(\mathrm{ck}_{t} ; \boldsymbol{a}_{\mathbf{2}} ; r_{2}\right)
\end{array}\right]=\operatorname{negl}(\kappa)
$$

A commitment scheme $\left(\mathcal{G}_{\text {com }}, \mathcal{C}\right.$ om $)$ is perfectly hiding in group $\mathbb{G}_{t}$, if for any positive integer $n=\operatorname{poly}(\kappa)$ and $\mathrm{ck}_{t} \in \mathcal{G}_{\text {com }}^{t}\left(1^{\kappa}, n\right)$ and any two messages $\boldsymbol{a}_{\mathbf{1}}, \boldsymbol{a}_{\mathbf{2}}$, the distributions $\mathcal{C o m}^{t}\left(\mathrm{ck}_{t} ; \boldsymbol{a}_{\mathbf{1}} ; \cdot\right)$ and $\mathcal{C} \mathrm{om}^{t}\left(\mathrm{ck}_{t} ; \boldsymbol{a}_{\mathbf{2}} ; \cdot\right)$ are equal.

A trapdoor commitment scheme has 3 additional efficient algorithms: (a) A trapdoor CRS generation algorithm inputs $t, n$ and $1^{\kappa}$, and outputs a CRS $\mathrm{ck}^{*}$ (that has the same distribution as $\mathcal{G}_{\mathrm{com}}^{t}\left(1^{\kappa}, n\right)$ ) and a trapdoor td, (b) a randomized trapdoor commitment algorithm takes $\mathrm{ck}^{*}$ and a randomizer $r$ as inputs, and outputs $\mathcal{C o m}^{t}\left(\mathrm{ck}^{*} ; \mathbf{0} ; r\right)$, and $(\mathrm{c})$ a trapdoor opening algorithm takes $\mathrm{ck}^{*}, \mathrm{td}$, $\boldsymbol{a}$ and $r$ as inputs, and outputs an $r^{\prime}$ such that $\mathcal{C o m}^{t}\left(\mathrm{ck}^{*} ; \mathbf{0} ; r\right)=\mathcal{C o m}^{t}\left(\mathrm{ck}^{*} ; \boldsymbol{a} ; r^{\prime}\right)$.

An extractable commitment scheme is a commitment scheme $\left(\mathcal{G}_{\text {com }}, \mathcal{C}\right.$ om $)$ with an additional extractor $\left(\operatorname{Extr}_{1}, \operatorname{Extr}_{2}\right)$ such that: $\operatorname{Extr}_{1}^{t}\left(1^{\kappa}\right)$ creates a CRS ck* (indistinguishable from the real CRS ck) and a trapdoor td, and $\operatorname{Extr}_{2}\left(\mathrm{ck}^{*}, \mathrm{td} ; A\right)$ returns $(a ; r)$ such that $A=\mathcal{C}$ om $($ ck; $a ; r)$, given that $A$ is a valid commitment. An extractable commitment scheme can only be computationally hiding.

We use the knowledge commitment scheme, defined in [15, as follows. 
CRS generation: Let $\Lambda$ be a $(n, \kappa)$-nice tuple with $n=\operatorname{poly}(\kappa)$. Let $\lambda_{0}=0$. Given a bilinear group generator $\mathcal{G}_{\mathrm{bp}}$, set gk $:=\left(p, \mathbb{G}_{1}, \mathbb{G}_{2}, \mathbb{G}_{T}, \hat{e}\right) \leftarrow \mathcal{G}_{\mathrm{bp}}\left(1^{\kappa}\right)$. Let $g_{1} \in \mathbb{G}_{1}$ and $g_{2} \in \mathbb{G}_{2}$ be generators, and choose random $\hat{\alpha}, x \leftarrow \mathbb{Z}_{p}$. Consider $t \in\{1,2\}$. The CRS is $\mathrm{ck}_{t} \leftarrow\left(\mathrm{gk} ;\left(g_{t, \lambda_{i}}, \hat{g}_{t, \lambda_{i}}\right)_{i \in\{0, \ldots, n\}}\right)$, where $g_{t, \lambda_{i}}=g_{t}^{x^{\lambda_{i}}}$, and $\hat{g}_{t, \lambda_{i}}=g_{t}^{\hat{\alpha} x^{\lambda_{i}}}$.

Commitment: To commit to $\boldsymbol{a}=\left(a_{1}, \ldots, a_{n}\right) \in \mathbb{Z}_{p}^{n}$, one chooses a random $r \leftarrow \mathbb{Z}_{p}$, and computes $\mathcal{C o m}^{t}\left(\mathrm{ck}_{t} ; \boldsymbol{a} ; r\right):=\left(g_{t}^{r} \cdot \prod_{i=1}^{n} g_{t, \lambda_{i}}^{a_{i}}, \hat{g}_{t}^{r} \cdot \prod_{i=1}^{n} \hat{g}_{t, \lambda_{i}}^{a_{i}}\right)$.

Let $t=1$. Fix a commitment key $\mathrm{ck}_{1}$ that in particular specifies $g_{2}, \hat{g}_{2} \in \mathbb{G}_{2}$. A commitment $(A, \hat{A}) \in \mathbb{G}_{1}^{2}$ is valid, if $\hat{e}\left(A, \hat{g}_{2}\right)=\hat{e}\left(\hat{A}, g_{2}\right)$. The case $t=2$ is dual.

According to [15, the knowledge commitment scheme is statistically hiding in group $\mathbb{G}_{t}$, and computationally binding in group $\mathbb{G}_{t}$ under the $\Lambda$-PSDL assumption in group $\mathbb{G}_{t}$. If the $\Lambda$-PKE assumption holds in group $\mathbb{G}_{t}$, then for any non-uniform PPT algorithm $\mathcal{A}$, that outputs some valid knowledge commitments, there exists a non-uniform PPT extractor $X_{\mathcal{A}}$ that, given as an input the input of $\mathcal{A}$ together with $\mathcal{A}$ 's random coins, extracts the contents of these commitments. The knowledge commitment scheme is also trapdoor, with the trapdoor being $\mathrm{td}=x$ : after trapdoor-committing $A \leftarrow \mathcal{C o m}^{t}(\mathrm{ck} ; \mathbf{0} ; r)=g_{t}^{r}$ for $r \leftarrow \mathbb{Z}_{p}$, the committer can open it to $\left(\boldsymbol{a} ; r-\sum_{i=1}^{n} a_{i} x^{\lambda_{i}}\right)$ for any $\boldsymbol{a}$.

Non-interactive Zero-Knowledge. Let $\mathcal{R}=\{(C, w)\}$ be an efficiently computable binary relation such that $|w|=\operatorname{poly}(|C|)$. Here, $C$ is a statement, and $w$ is a witness. Let $\mathcal{L}=\{C: \exists w,(C, w) \in \mathcal{R}\}$ be an NP-language. Let $n=|C|$ be a fixed input length. For fixed $n$, we have a relation $\mathcal{R}_{n}$ and a language $\mathcal{L}_{n}$. A non-interactive argument for $\mathcal{R}$ consists of the next PPT algorithms: a common reference string (CRS) generator $\mathcal{G}_{\text {crs }}$, a prover $\mathcal{P}$, and a verifier $\mathcal{V}$. For crs $\leftarrow \mathcal{G}_{\mathrm{crs}}\left(1^{\kappa}, n\right), \mathcal{P}(\mathrm{crs} ; C, w)$ produces an argument $\psi$. The verifier $\mathcal{V}(\mathrm{crs} ; C, \psi)$ outputs either 1 (accept) or 0 (reject).

A non-interactive argument $\left(\mathcal{G}_{\mathrm{crs}}, \mathcal{P}, \mathcal{V}\right)$ is perfectly complete, if for all values $n=\operatorname{poly}(\kappa)$, all crs $\leftarrow \mathcal{G}_{\text {crs }}\left(1^{\kappa}, n\right)$ and all $(C, w) \in \mathcal{R}_{n}, \mathcal{V}(\mathrm{crs} ; C, \mathcal{P}(\mathrm{crs} ; C, w))=1$. A non-interactive argument $\left(\mathcal{G}_{\mathrm{crs}}, \mathcal{P}, \mathcal{V}\right)$ is computationally (adaptively) sound, if for all non-uniform PPT adversaries $\mathcal{A}$ and all $n=\operatorname{poly}(\kappa)$,

$$
\operatorname{Pr}\left[\mathrm{crs} \leftarrow \mathcal{G}_{\mathrm{crs}}\left(1^{\kappa}, n\right),(C, \psi) \leftarrow \mathcal{A}(\mathrm{crs}): C \notin \mathcal{L} \wedge \mathcal{V}(\mathrm{crs} ; C, \psi)=1\right]=\operatorname{negl}(\kappa)
$$

A non-interactive argument $\left(\mathcal{G}_{\mathrm{crs}}, \mathcal{P}, \mathcal{V}\right)$ is perfectly witness-indistinguishable, if (given that there are several possible witnesses) it is impossible to tell which witness the prover used. That is, for all $n=\operatorname{poly}(\kappa)$, if $\operatorname{crs} \in \mathcal{G}_{\mathrm{crs}}\left(1^{\kappa}, n\right)$ and $\left(\left(C, w_{0}\right),\left(C, w_{1}\right)\right) \in \mathcal{R}_{n}^{2}$, then the distributions $\mathcal{P}\left(\mathrm{crs} ; C, w_{0}\right)$ and $\mathcal{P}\left(\mathrm{crs} ; C, w_{1}\right)$ are equal. $\left(\mathcal{G}_{\mathrm{crs}}, \mathcal{P}, \mathcal{V}\right)$ is perfectly zero-knowledge, if there exists a polynomialtime simulator $\mathcal{S}=\left(\mathcal{S}_{1}, \mathcal{S}_{2}\right)$, such that for all stateful interactive non-uniform PPT adversaries $\mathcal{A}$ and $n=\operatorname{poly}(\kappa)$,

$$
\operatorname{Pr}\left[\begin{array}{l}
\mathrm{crs} \leftarrow \mathcal{G}_{\mathrm{crs}}\left(1^{\kappa}, n\right), \\
(C, w) \leftarrow \mathcal{A}(\mathrm{crs}), \\
\psi \leftarrow \mathcal{P}(\mathrm{crs} ; C, w): \\
(C, w) \in \mathcal{R}_{n} \wedge \mathcal{A}(\psi)=1
\end{array}\right]=\operatorname{Pr}\left[\begin{array}{l}
(\mathrm{crs}, \mathrm{td}) \leftarrow \mathcal{S}_{1}\left(1^{\kappa}, n\right), \\
(C, w) \leftarrow \mathcal{A}(\mathrm{crs}), \\
\psi \leftarrow \mathcal{S}_{2}(\mathrm{crs}, C, \mathrm{td}): \\
(C, w) \in \mathcal{R}_{n} \wedge \mathcal{A}(\psi)=1
\end{array}\right]
$$


System parameters: Let $n=\operatorname{poly}(\kappa)$. Let $\Lambda=\left\{\lambda_{i}: i \in[n]\right\}$ be a progression-free set of odd integers, such that $\lambda_{i+1}>\lambda_{i}>0$. Denote $\lambda_{0}:=0$. Let $\hat{\Lambda}:=\{0\} \cup \Lambda \cup 2^{\wedge} \Lambda$.

CRS generation $\mathcal{G}_{\mathrm{crs}}\left(1^{\kappa}\right)$ : Let gk $:=\left(p, \mathbb{G}_{1}, \mathbb{G}_{2}, \mathbb{G}_{T}, \hat{e}\right) \leftarrow \mathcal{G}_{\mathrm{bp}}\left(1^{\kappa}\right)$. Let $\hat{\alpha}, x \leftarrow \mathbb{Z}_{p}$. Let $g_{1} \leftarrow \mathbb{G}_{1} \backslash\{1\}$ and $g_{2} \leftarrow \mathbb{G}_{2} \backslash\{1\}$. Denote $g_{t \ell} \leftarrow g_{t}^{x^{\ell}}$ and $\hat{g}_{t \ell} \leftarrow g_{t}^{\hat{\alpha} x^{\ell}}$ for $t \in\{1,2\}$ and $\ell \in\{0\} \cup \hat{\Lambda}$. Let $D \leftarrow \prod_{i=1}^{n} g_{2, \lambda_{i}}$. The CRS is crs $\leftarrow$ $\left(\mathrm{gk} ;\left(g_{1 \ell}, \hat{g}_{1 \ell}\right)_{\ell \in\{0\} \cup \Lambda},\left(g_{2 \ell}, \hat{g}_{2 \ell}\right)_{\ell \in \hat{\Lambda}}, D\right)$. Let $\widehat{c k}_{1} \leftarrow\left(\mathrm{gk} ;\left(g_{1 \ell}, \hat{g}_{1 \ell}\right)_{\ell \in\{0\} \cup \Lambda}\right)$.

Common inputs: $\left(A, \hat{A}, B, \hat{B}, B_{2}, C, \hat{C}\right)$, where $(A, \hat{A}) \leftarrow \mathcal{C o m}^{1}\left(\widehat{c k}_{1} ; \boldsymbol{a} ; r_{a}\right),(B, \hat{B}) \leftarrow$ $\mathcal{C o m}^{1}\left(\widehat{c k}_{1} ; \boldsymbol{b} ; r_{b}\right), B_{2} \leftarrow g_{2}^{r_{b}} \cdot \prod_{i=1}^{n} g_{2, \lambda_{i}}^{b_{i}},(C, \hat{C}) \leftarrow \mathcal{C o m}^{1}\left(\widehat{c k}_{1} ; \boldsymbol{c} ; r_{c}\right)$, s.t. $a_{i} b_{i}=c_{i}$ for $i \in[n]$.

Argument generation $\mathcal{P}_{\times}\left(\operatorname{crs} ;\left(A, \hat{A}, B, \hat{B}, B_{2}, C, \hat{C}\right),\left(\boldsymbol{a}, r_{a}, \boldsymbol{b}, r_{b}, \boldsymbol{c}, r_{c}\right)\right)$ : Let $I_{1}(\ell):=\left\{(i, j): i, j \in[n] \wedge j \neq i \wedge \lambda_{i}+\lambda_{j}=\ell\right\}$. For $\ell \in 2^{\wedge} \Lambda$, the prover sets $\mu_{\ell} \leftarrow \sum_{(i, j) \in I_{1}(\ell)}\left(a_{i} b_{j}-c_{i}\right)$. He sets $\psi \leftarrow g_{2}^{r_{a} r_{b}} \cdot \prod_{i=1}^{n} g_{2, \lambda_{i}}^{r_{a} b_{i}+r_{b} a_{i}-r_{c}} \cdot \prod_{\ell \in 2^{-} \Lambda} g_{2 \ell}^{\mu_{\ell}}$, and $\hat{\psi} \leftarrow \hat{g}_{2}^{r_{a} r_{b}} \cdot \prod_{i=1}^{n} \hat{g}_{2, \lambda_{i}}^{r_{a} b_{i}+r_{b} a_{i}-r_{c}} \cdot \prod_{\ell \in 2^{-} \Lambda} \hat{g}_{2 \ell}^{\mu_{\ell}}$. He sends $\psi^{\times} \leftarrow(\psi, \hat{\psi}) \in \mathbb{G}_{2}^{2}$ to the verifier as the argument.

Verification $\mathcal{V}_{\times}\left(\operatorname{crs} ;\left(A, \hat{A}, B, \hat{B}, B_{2}, C, \hat{C}\right), \psi^{\times}\right)$: accept $\quad$ iff $\quad \hat{e}\left(A, B_{2}\right) / \hat{e}(C, D) \quad=$ $\hat{e}\left(g_{1}, \psi\right)$ and $\hat{e}\left(g_{1}, \hat{\psi}\right)=\hat{e}\left(\hat{g}_{1}, \psi\right)$.

Protocol 1. Hadamard product argument $\llbracket(A, \hat{A}) \rrbracket \circ \llbracket\left(B, \hat{B}, B_{2}\right) \rrbracket=\llbracket(C, \hat{C}) \rrbracket$

Here, $\mathrm{td}$ is the simulation trapdoor. $\left(\mathcal{G}_{\mathrm{crs}}, \mathcal{P}, \mathcal{V}\right)$ is computationally zero-knowledge if these two probabilities are computationally indistinguishable.

\section{Groth-Lipmaa Arguments}

In this section, we describe two of our building-blocks, an Hadamard product argument and a (known) permutation argument. In both cases, Groth [8] proposed efficient (weakly) sound and non-interactive witness-indistinguishable (NIWI) arguments that were further refined by Lipmaa [15, who used the theory of progression-free sets to optimize Groth's arguments. Since [15] is very new, we will give here a full description of Lipmaa's NIWI arguments. We refer to [15] (and its full version, [14]) for details.

\subsection{Hadamard Product Argument}

Let $\left(\mathcal{G}_{\text {com }}, \mathcal{C}\right.$ om $)$ be the knowledge commitment scheme. An Hadamard product of two vectors $\boldsymbol{a}$ and $\boldsymbol{b}$ is equal to their entrywise product vector $\boldsymbol{c}$, that is, $c_{j}=a_{j} \cdot b_{j}$ for $j \in[n]$. In an Hadamard product argument, the prover aims to convince the verifier that for given three commitments $(A, \hat{A}),(B, \hat{B})$ and $(C, \hat{C})$, he knows how to open them as $(A, \hat{A})=\mathcal{C}^{1}\left(\mathrm{ck} ; \boldsymbol{a} ; r_{a}\right),(B, \hat{B})=\mathcal{C o m}^{1}\left(\mathrm{ck} ; \boldsymbol{b} ; r_{b}\right)$, and $(C, \hat{C})=\mathcal{C o m}^{1}\left(\mathrm{ck} ; \boldsymbol{c} ; r_{c}\right)$, such that $c_{j}=a_{j} \cdot b_{j}$ for $j \in[n]$. Prot. 1 has a full description of Lipmaa's Hadamard product argument $\llbracket(A, \hat{A}) \rrbracket \circ \llbracket\left(B, \hat{B}, B_{2}\right) \rrbracket=$ $\llbracket(C, \hat{C}) \rrbracket$, where $B_{2}$ is the equivalent of $B$ in $\mathbb{G}_{2}: B_{2} \leftarrow g_{2}^{r_{b}} \cdot \prod_{i=1}^{n} g_{2, \lambda_{i}}^{b_{i}}$.

Fact 2 (Lipmaa [15]). The above Hadamard product argument is perfectly complete and perfectly witness-indistinguishable. If the bilinear group generator 
$\mathcal{G}_{\mathrm{bp}}$ is $\hat{\Lambda}-P S D L$ secure, then a non-uniform PPT adversary has negligible chance of outputting inp ${ }^{\times} \leftarrow\left(A, \hat{A}, B, \hat{B}, B_{2}, C, \hat{C}\right)$ and an accepting argument $\psi^{\times} \leftarrow$ $(\psi, \hat{\psi})$ together with an opening witness $w^{\times} \leftarrow\left(\boldsymbol{a}, r_{a}, \boldsymbol{b}, r_{b}, \boldsymbol{c}, r_{c},\left(f_{s}^{\prime}\right)_{s \in \hat{\Lambda}}\right)$, such that $(A, \hat{A})=\mathcal{C o m}^{1}\left(\widehat{\mathrm{ck}}_{1} ; \boldsymbol{a} ; r_{a}\right),(B, \hat{B})=\mathcal{C o m}^{1}\left(\widehat{c k}_{1} ; \boldsymbol{b} ; r_{b}\right), B_{2}=g_{2}^{r_{b}} \cdot \prod_{i=1}^{n} g_{2 i}^{b_{i}}$, $(C, \hat{C})=\mathcal{C o m}^{1}\left(\widehat{c k}_{1} ; c ; r_{c}\right),(\psi, \hat{\psi})=\left(g_{2}^{\sum_{s \in \hat{A}} f_{s}^{\prime} x^{s}}, \hat{g}_{2}^{\sum_{s \in \hat{\Lambda}} f_{s}^{\prime} x^{s}}\right)$, and for some $i \in[n], a_{i} b_{i} \neq c_{i}$.

For the product argument to be useful in more complex arguments, we must also assume that the verifier there additionally verifies that $\hat{e}\left(A, \hat{g}_{2}\right)=\hat{e}\left(\hat{A}, g_{2}\right)$, $\hat{e}\left(B, \hat{g}_{2}\right)=\hat{e}\left(\hat{B}, g_{2}\right), \hat{e}\left(g_{1}, B_{2}\right)=\hat{e}\left(B, g_{2}\right)$, and $\hat{e}\left(C, \hat{g}_{2}\right)=\hat{e}\left(\hat{C}, g_{2}\right)$. Note that $\left(f_{s}^{\prime}\right)_{s \in \hat{\Lambda}}$ is the opening of $(\psi, \hat{\psi})$.

Fact 3 (Lipmaa [15]). For any $n>0$ and $y=n^{1+o(1)}$, let $\Lambda \subset[y]$ be a progression-free set of odd integers from Fact 1 , such that $|\Lambda|=n$. The communication (argument size) of the Hadamard product argument is 2 elements from $\mathbb{G}_{2}$. The prover's computational complexity is $\Theta\left(n^{2}\right)$ scalar multiplications in $\mathbb{Z}_{p}$ and $n^{1+o(1)}$ exponentiations in $\mathbb{G}_{2}$. The verifier's computational complexity is dominated by 5 bilinear pairings. The CRS consists of $n^{1+o(1)}$ group elements.

Finally, if $\boldsymbol{a}, \boldsymbol{b}$ and $\boldsymbol{c}$ are Boolean vectors then the prover's computational complexity is $\Theta\left(n^{2}\right)$ scalar additions in $\mathbb{Z}_{p}$ and $n^{1+o(1)}$ exponentiations in $\mathbb{G}$ [15.

\subsection{Permutation Argument}

In a permutation argument, the prover aims to convince the verifier that for given permutation $\varrho \in S_{n}$ and two commitments $(A, \tilde{A})$ and $(B, \tilde{B})$, he knows how to open them as $(A, \tilde{A})=\mathcal{C}{ }^{1}\left(\mathrm{ck} ; \boldsymbol{a} ; r_{a}\right)$ and $(B, \tilde{B})=\mathcal{C o m}^{1}\left(\mathrm{ck} ; \boldsymbol{b} ; r_{b}\right)$, such that $b_{j}=a_{\varrho(j)}$ for $j \in[n]$. We denote this non-interactive argument by $\varrho(\llbracket(A, \tilde{A}) \rrbracket)=\llbracket\left(B, \tilde{B}, B_{2}\right) \rrbracket$, where $B_{2}$ is again the equivalent of $B$ in $\mathbb{G}_{2}$. As in the case of the Hadamard product argument, we describe a version of the argument due to [15. See Prot. 2.

Let $T_{\Lambda}(i, \varrho):=\left|\left\{j \in[n]: 2 \lambda_{\varrho(i)}+\lambda_{j}=2 \lambda_{\varrho(j)}+\lambda_{i}\right\}\right|$, clearly $T_{\Lambda}(i, \varrho) \geq 1$. One proves that $a_{\varrho(i)}=b_{i}$ for $i \in[n]$ by using a subargument that shows that for separately committed $a_{i}^{*}, a_{\varrho(i)}^{*}=T_{\Lambda}(i, \varrho) \cdot b_{i}$ for $i \in[n]$. Showing in addition that $a_{i}^{*}=T_{\Lambda}\left(\varrho^{-1}(i), \varrho\right) \cdot a_{i}$ (which is equivalent to $\left.a_{\varrho(i)}^{*}=T_{\Lambda}(i, \varrho) \cdot a_{\varrho(i)}\right)$, one obtains that $a_{\varrho(i)}=b_{i}$ for $i \in[n]$. We only consider the case where $\varrho$ is fixed and thus the element $E_{\varrho}$ can be put to the CRS. We also use the fact that $\hat{\Lambda} \cup \tilde{\Lambda}=\{0\} \cup \tilde{\Lambda}$, where $\tilde{\Lambda}$ is defined in Prot. 2

We denote the full permutation argument by $\varrho(\llbracket(A, \tilde{A}) \rrbracket)=\llbracket(B, \hat{B}, \tilde{B}) \rrbracket$.

Fact 4 (Lipmaa [15]). The above permutation argument is perfectly complete and perfectly witness-indistinguishable. If the bilinear group generator $\mathcal{G}_{\mathrm{bp}}$ is $\widetilde{\Lambda}-P S D L$ secure, then a non-uniform PPT adversary has negligible chance of outputting inp perm $\leftarrow(A, \tilde{A}, B, \hat{B}, \tilde{B}, \varrho)$ and an accepting argument $\psi^{\text {perm }} \leftarrow$ $\left(A^{*}, \hat{A}^{*}, \psi^{\times}, \hat{\psi}^{\times}, \psi^{\varrho}, \widetilde{\psi}^{\varrho}\right)$ together with an opening witness

$$
w^{\text {perm }} \leftarrow\left(\boldsymbol{a}, r_{a}, \boldsymbol{b}, r_{b}, \boldsymbol{a}^{*}, r_{a^{*}},\left(f_{(\times, \ell)}^{\prime}\right)_{\ell \in \hat{\Lambda}},\left(f_{(\varrho, \ell)}^{\prime}\right)_{\ell \in \tilde{\Lambda}}\right)
$$


System parameters: Same as in Prot. 1, but let $\tilde{\Lambda}:=\Lambda \cup\left\{2 \lambda_{k}-\lambda_{j}\right\}_{i, k \in[n]} \cup 2^{\wedge} \Lambda \cup$ $\left(\left\{2 \lambda_{k}+\lambda_{i}-\lambda_{j}\right\}_{i, j, k \in[n] \wedge i \neq j} \backslash 2 \cdot \Lambda\right)$.

CRS generation $\mathcal{G}_{\text {crs }}\left(1^{\kappa}\right)$ : Let gk $:=\left(p, \mathbb{G}_{1}, \mathbb{G}_{2}, \mathbb{G}_{T}, \hat{e}\right) \leftarrow \mathcal{G}_{\mathrm{bp}}\left(1^{\kappa}\right)$. Let $\hat{\alpha}, \tilde{\alpha}, x \leftarrow \mathbb{Z}_{p}$. Let $g_{1} \leftarrow \mathbb{G}_{1} \backslash\{1\}$ and $g_{2} \leftarrow \mathbb{G}_{2} \backslash\{1\}$. Let $\hat{g}_{t} \leftarrow \hat{g}_{t}^{\hat{\alpha}}$ and $\tilde{g}_{t} \leftarrow \tilde{g}_{t}^{\tilde{\alpha}}$ for $t \in\{1,2\}$. Denote $g_{t \ell} \leftarrow g_{t}^{x^{\ell}}, \hat{g}_{t \ell} \leftarrow \hat{g}_{t}^{x^{\ell}}$, and $\tilde{g}_{t \ell} \leftarrow \tilde{g}_{t}^{x^{\ell}}$ for $t \in\{1,2\}$ and $\ell \in\{0\} \cup \tilde{\Lambda}$. Let $(D, \tilde{D}) \leftarrow\left(\prod_{i=1}^{n} g_{2, \lambda_{i}}, \prod_{i=1}^{n} \tilde{g}_{2, \lambda_{i}}\right)$. The CRS is

$$
\operatorname{crs} \leftarrow\left(\mathrm{gk} ;\left(g_{1 \ell}, \hat{g}_{1 \ell}, \tilde{g}_{1 \ell}\right)_{\ell \in\{0\} \cup \Lambda},\left(g_{2 \ell}\right)_{\ell \in\{0\} \cup \tilde{\Lambda}},\left(\hat{g}_{2 \ell}\right)_{\ell \in \hat{\Lambda}},\left(\tilde{g}_{2 \ell}\right)_{\ell \in \tilde{\Lambda}}, D, \tilde{D}\right) .
$$

Let $\widehat{\mathrm{ck}}_{1} \leftarrow\left(\mathrm{gk} ;\left(g_{1 \ell}, \hat{g}_{1 \ell}\right)_{\ell \in\{0\} \cup \Lambda}\right), \widetilde{\mathrm{ck}}_{1} \leftarrow\left(\mathrm{gk} ;\left(g_{1 \ell}, \tilde{g}_{1 \ell}\right)_{\ell \in\{0\} \cup \Lambda}\right)$.

Common inputs: $(A, \tilde{A}, B, \hat{B}, \tilde{B}, \varrho)$, where $\varrho \in S_{n},(A, \tilde{A}) \leftarrow \mathcal{C o m}^{1}\left(\widetilde{c k}_{1} ; \boldsymbol{a} ; r_{a}\right)$, $(B, \hat{B}) \leftarrow \mathcal{C o m}^{1}\left(\widehat{\mathrm{ck}}_{1} ; \boldsymbol{b} ; r_{b}\right)$, and $(B, \tilde{B}) \leftarrow \mathcal{C o m}^{1}\left(\widetilde{\mathrm{ck}_{1}} ; \boldsymbol{b} ; r_{b}\right)$, s.t. $b_{j}=a_{\varrho(j)}$ for $j \in[n]$. Argument generation $\mathcal{P}_{\text {perm }}\left(\mathrm{crs} ;(A, \tilde{A}, B, \hat{B}, \tilde{B}, \varrho),\left(\boldsymbol{a}, r_{a}, \boldsymbol{b}, r_{b}\right)\right)$ :

1. Let $\left(T^{*}, \hat{T}^{*}, T_{2}^{*}\right) \leftarrow\left(\prod_{i=1}^{n} g_{1, \lambda_{i}}^{T_{\Lambda}\left(\varrho^{-1}(i), \varrho\right)}, \prod_{i=1}^{n} \hat{g}_{1, \lambda_{i}}^{T_{\Lambda}\left(\varrho^{-1}(i), \varrho\right)}, \prod_{i=1}^{n} g_{2, \lambda_{i}}^{T_{\Lambda}\left(\varrho^{-1}(i), \varrho\right)}\right)$.

2. Let $r_{a^{*}} \leftarrow \mathbb{Z}_{p},\left(A^{*}, \hat{A}^{*}\right) \leftarrow \operatorname{Com}_{1}\left(\widehat{c k}_{1} ; T_{\Lambda}\left(\varrho^{-1}(1), \varrho\right) \cdot a_{1}, \ldots, T_{\Lambda}\left(\varrho^{-1}(n), \varrho\right)\right.$ $\left.a_{n} ; r_{a^{*}}\right)$. Create an argument $\psi^{\times}$for $\llbracket(A, \hat{A}) \rrbracket \circ \llbracket\left(T^{*}, \hat{T}^{*}, T_{2}^{*}\right) \rrbracket=\llbracket\left(A^{*}, \hat{A}^{*}\right) \rrbracket$.

3. Let $\tilde{\Lambda}_{\varrho}^{\prime}:=2^{\wedge} \Lambda \cup\left(\left\{2 \lambda_{\varrho(j)}+\lambda_{i}-\lambda_{j}: i, j \in[n] \wedge i \neq j\right\} \backslash 2 \cdot \Lambda\right) \subset\left\{-\lambda_{n}+1, \ldots, 3 \lambda_{n}\right\}$.

4. For $\ell \in \tilde{\Lambda}_{\varrho}^{\prime}, I_{1}(\ell)$ as in Prot. 1 and $I_{2}(\ell):=\left\{(i, j): i, j \in[n] \wedge j \neq i \wedge 2 \lambda_{\varrho(i)}+\right.$ $\left.\lambda_{j} \neq \lambda_{i}+2 \lambda_{\varrho(j)} \wedge 2 \lambda_{\varrho(j)}+\lambda_{i}-\lambda_{j}=\ell\right\}$, set $\mu_{\varrho, \ell} \leftarrow \sum_{(i, j) \in I_{1}(\ell)} a_{i}^{*}-\sum_{(i, j) \in I_{2}(\ell)} b_{i}$.

5. Let $\left(E_{\varrho}, \tilde{E}_{\varrho}\right) \leftarrow\left(\prod_{i=1}^{n} g_{2,2 \lambda_{\varrho(i)}}-\lambda_{i}, \prod_{i=1}^{n} \tilde{g}_{2,2 \lambda_{\varrho(i)}}-\lambda_{i}\right)$.

6. Let $\psi^{\varrho} \leftarrow D^{r_{a}^{*}} \cdot E_{\varrho}^{-r_{b}} \cdot \prod_{\ell \in \tilde{\Lambda}_{\varrho}^{\prime}} g_{2 \ell}^{\mu_{\varrho}, \ell}, \tilde{\psi}^{\varrho} \leftarrow \tilde{D}^{r_{a}^{*}} \cdot \tilde{E}_{\varrho}^{-r_{b}} \cdot \prod_{\ell \in \tilde{\Lambda}_{\varrho}^{\prime}} \tilde{g}_{2 \ell}^{\mu_{\varrho}, \ell}$,

Send $\psi^{\text {perm }} \leftarrow\left(A^{*}, \hat{A}^{*}, \psi^{\times}, \psi^{\varrho}, \tilde{\psi}^{\varrho}\right) \in \mathbb{G}_{1}^{2} \times \mathbb{G}_{2}^{4}$ to the verifier as the argument.

Verification $\mathcal{V}_{\text {perm }}\left(\operatorname{crs} ;(A, \tilde{A}, B, \hat{B}, \tilde{B}, \varrho), \psi^{\text {perm }}\right)$ : Let $E_{\varrho}$ and $\left(T^{*}, \hat{T}^{*}, T_{2}^{*}\right)$ be computed as in $\mathcal{P}_{\text {perm. }}$. If $\psi^{\times}$verifies, $\hat{e}\left(A^{*}, D\right) / \hat{e}\left(B, E_{\varrho}\right)=\hat{e}\left(g_{1}, \psi^{\varrho}\right), \hat{e}\left(A^{*}, \hat{g}_{2}\right)=$

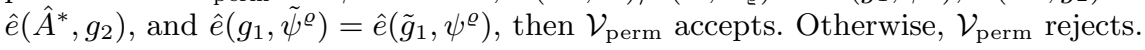

Protocol 2. Permutation argument $\varrho(\llbracket(A, \tilde{A}) \rrbracket)=\llbracket(B, \tilde{B}) \rrbracket$ from $\llbracket 15]$

such that $(A, \tilde{A})=\operatorname{Com}^{1}\left(\widetilde{c k}_{1} ; \boldsymbol{a} ; r_{a}\right),(B, \hat{B})=\operatorname{Com}^{1}\left(\widehat{c k}_{1} ; \boldsymbol{b} ; r_{b}\right)$, $(B, \tilde{B})=\mathcal{C o m}^{1}\left(\widetilde{c k}_{1} ; \boldsymbol{b} ; r_{b}\right),\left(A^{*}, \hat{A}^{*}\right)=\mathcal{C o m}^{1}\left(\widehat{c k}_{1} ; \boldsymbol{a}^{*} ; r_{a^{*}}\right),\left(\psi^{\times}, \hat{\psi}^{\times}\right)=$ $\left(g_{2}^{\sum_{\ell \in \hat{\Lambda}} f_{(\times, \ell)}^{\prime}}, \hat{g}_{2}^{\sum_{\ell \in \hat{\Lambda}} f_{(\times, \ell)}^{\prime}}\right),\left(\psi^{\varrho}, \hat{\psi}^{\varrho}\right)=\left(g_{2}^{\sum_{\ell \in \tilde{\Lambda}} f_{(\varrho, \ell)}^{\prime}}, \tilde{g}_{2}^{\sum_{\ell \in \tilde{\Lambda}} f_{(\varrho, \ell)}^{\prime}}\right), \quad a_{i}^{*}=$ $T_{\Lambda}\left(\varrho^{-1}(i), \varrho\right) \cdot a_{i}$ (for $\left.i \in[n]\right)$, and for some $i \in[n]$, $a_{\varrho(i)} \neq b_{i}$.

For the permutation argument to be useful in more complex arguments, we must also assume that the verifier there verifies that $\hat{e}\left(\tilde{A}, g_{2}\right)=\hat{e}\left(A, \tilde{g}_{2}\right), \hat{e}\left(\hat{B}, g_{2}\right)=$ $\hat{e}\left(B, \hat{g}_{2}\right)$, and $\hat{e}\left(\tilde{B}, g_{2}\right)=\hat{e}\left(B, \tilde{g}_{2}\right)$.

Fact 5 (Lipmaa [15]). The permutation argument has a CRS of length $n^{1+o(1)}$ and communication of 4 group elements. The prover's computational complexity is $\Theta\left(n^{2}\right)$ scalar additions in $\mathbb{Z}_{p}$ and $n^{1+o(1)}$ exponentiations in $\mathbb{G}$. The verifier's computational complexity is dominated by 12 bilinear pairings.

\section{Breaking the COCOON 2009 Range Proof}

In [21, the authors proposed a non-interactive range proof. In what follows, we show that their argument is not secure. 
Their goal is to prove that a committed secret $w$ is in some range $[a, b]$. To do so they prove that both $w-a$ and $b-w$ are non-negative by making use of Lagrange theorem stating that any non-negative integer can be decomposed as the sum of four squares. Hence,

$$
w-a=\sum_{j=1}^{4} w_{1 j}^{2} \quad \text { and } \quad b-w=\sum_{j=1}^{4} w_{2 j}^{2},
$$

for some $w_{i j}$. The range proof of [21] is based on (symmetric) bilinear groups of composite order, i.e., on bilinear groups $\left(n, \mathbb{G}_{,} \mathbb{G}_{T}, \hat{e}\right)$, where $n=p q$. To commit to a message $w$, the committer picks a random 1$] \in \mathbb{Z}_{q}$ and computes $C=g^{w} u^{r}$, where $g$ is a random generator of $\mathbb{G}$ (of order $n$ ), and $u$ is a random generator of subgroup $\mathbb{G}_{q}$ (of order $q$ ). Given $C, w$ is uniquely determined in $\mathbb{Z}_{p}$ as $C^{q}=g^{w q}$.

In their range proof, the prover finds the witnesses $w_{i j}$ in Eq. (1) and outputs a proof $\psi=\left(\left\{C_{1 j}, C_{2 j}\right\}_{j \in[4]}, C_{w}, \varphi_{1}, \varphi_{2}\right)$, where $C_{w} \equiv g^{w} u^{r_{w}} \in \mathbb{G}$, $C_{i j} \equiv g^{w_{i j}} u^{r_{i j}} \in \mathbb{G}$ for $i \in[2]$ and $j \in[4], \varphi_{1} \equiv g^{-r_{w}+2 \sum_{j=1}^{4} r_{1 j} w_{1 j}} \cdot u^{\sum_{j=1}^{4} r_{1 j}^{2}} \in \mathbb{G}$, $\varphi_{2} \equiv g^{r_{w}+2 \sum_{j=1}^{4} r_{2 j} w_{2 j}} \cdot u^{\sum_{j=1}^{4} r_{2 j}^{2}} \in \mathbb{G}$. The verifier checks whether $\hat{e}\left(g^{a} C_{w}^{-1}, g\right)$. $\prod_{j=1}^{4} e\left(C_{1 j}, C_{1 j}\right)=\hat{e}\left(u, \varphi_{1}\right)$ and $\hat{e}\left(C_{w} g^{-b}, g\right) \cdot \prod_{j=1}^{4} \hat{e}\left(C_{2 j}, C_{2 j}\right)=\hat{e}\left(u, \varphi_{2}\right)$.

Now assume that a malicious prover $P^{\star}$ picks an integer $w^{*} \in\{0, \ldots, p-1\} \backslash$ $[a, b]$. We have that either $w^{*}-a$ or $b-w^{*}$ is negative as an integer. Suppose $b-w^{*}<0$, then $P^{\star}$ chooses $\left\{w_{2 j}^{*}\right\}_{j \in[4]}$ such that $n+\left(b-w^{*}\right)=\sum_{j=1}^{4}\left(w_{2 j}^{*}\right)^{2}$, sets $C_{w} \leftarrow g^{w^{*}} u^{r_{w}}, C_{2 j} \leftarrow g^{w_{2 j}^{*}} u^{r_{2 j}}, \varphi_{1}$ as above, and $\varphi_{2} \leftarrow g^{r_{w}+2 \cdot \sum_{j=1}^{4} r_{2 j} w_{2 j}^{*}}$. $u^{\sum_{j=1}^{4} r_{2 j}^{2}}$. Let $u=g^{\alpha}$ for some $\alpha$. It is easy to see that the second verification equation still holds:

$$
\begin{aligned}
\hat{e}\left(C_{w} g^{-b}, g\right) & \cdot \prod_{j=1}^{4} \hat{e}\left(C_{2 j}, C_{2 j}\right)=\hat{e}(g, g)^{\left(w^{*}-b\right)+\alpha r_{w}+\sum_{j=1}^{4}\left(w_{2 j}^{*}+\alpha r_{2 j}\right)^{2}} \\
= & \hat{e}(g, g)^{\left(w^{*}-b\right)+\alpha r_{w}+\sum_{j=1}^{4}\left(w_{2 j}^{*}\right)^{2}+\sum_{j=1}^{4} \alpha^{2} r_{2 j}^{2}+2 \sum_{j=1}^{4} \alpha r_{2 j} w_{2 j}^{*}} \\
= & \hat{e}(g, g)^{\alpha \cdot\left(r_{w}+2 \sum_{j=1}^{4} r_{2 j} w_{2 j}^{*}+\alpha \cdot \sum_{j=1}^{4} r_{2 j}^{2}\right)}=\hat{e}\left(u, \varphi_{2}\right) .
\end{aligned}
$$

We have successfully constructed a polynomial time adversary who can always break the scheme. Therefore, the NIZK range proof in 21] is not sound.

\section{New Subargument for Correct Encryption}

In the new range proof of Sect. 6, we need a subargument that if $\left(A_{c}, \bar{A}_{c}\right)$ is a knowledge-commitment of some $a$ (with $n=1$ and some randomness $r$ ), and $\left(A_{g}, A_{f}, A_{h}\right)$ is a BBS ciphertext of some $a^{\prime}$, then $a=a^{\prime}$. That is, $A_{c}=g_{1}^{r} g_{1, \lambda_{1}}^{a}$ and $\left(A_{g}, A_{f}, A_{h}\right)=\left(g_{1}^{r_{f}+r_{h}+a}, f^{r_{f}}, h^{r_{h}}\right)$ for randomness $\left(r_{f}, r_{h}\right)$ and public key $(f, h)$. (The generator $g_{1, \lambda_{1}}$ is required in Sect. 6.)

\footnotetext{
${ }^{1}$ In [21], the scheme uses $r \in \mathbb{Z}_{n}$ to facilitate their security proof (crs switching).
} 
We will construct this argument in the current section, by combining ideas from [1] and 815]. Intuitively, for every multi-exponentiation $h_{1}^{a_{1}} \ldots h_{m}^{a_{m}}=t$ that we want to prove, we write down a verification equation $\hat{e}\left(h_{1}, \mathcal{C}\right.$ om $\left.\left(a_{1}\right)\right)$. $\cdots \hat{e}\left(h_{m}, \operatorname{Com}\left(a_{m}\right)\right)=\hat{e}\left(\psi, g_{2}\right) \hat{e}(t, \mathcal{C o m}(1))$, where $\psi$ "compensates" for the fact that $\operatorname{Com}\left(a_{m}\right)$ are probabilistic commitments. In addition, we use knowledge commitments (though for small values 0 or 1 of $n$ ) so that one can extract all committed values. Since the argument uses three committed values $\left(a, r_{f}\right.$ and $r_{h}$ ) and three equations, according to Fig. 6 of [10] (the full version of [11]), the corresponding pure Groth-Sahai argument will have length of 15 group elements. Our argument has the same length, but is computationally more efficient.

System parameters: An $(n, \kappa)$-nice tuple $\Lambda=\left(\lambda_{1}, \ldots, \lambda_{n}\right)$.

Common reference string generation $\mathcal{G}_{\mathrm{crs}}\left(1^{\kappa}\right)$ : Set

$$
\text { gk }:=\left(p, \mathbb{G}_{1}, \mathbb{G}_{2}, \mathbb{G}_{t}, \hat{e}\right) \leftarrow \mathcal{G}_{\mathrm{bp}}\left(1^{\kappa}\right)
$$

Generate random $\alpha_{g}, \alpha_{f}, \alpha_{h}, \bar{\alpha}, \alpha_{g / c}, x \leftarrow \mathbb{Z}_{p}$. Let $g_{1} \leftarrow \mathbb{G}_{1} \backslash\{1\}$ and $g_{2} \leftarrow$ $\mathbb{G}_{2} \backslash\{1\}$. Denote $g_{1, \lambda_{1}} \leftarrow g_{1}^{x^{\lambda_{1}}}, g_{2, \lambda_{1}} \leftarrow g_{2}^{x^{\lambda_{1}}}, \stackrel{\circ}{g}_{1} \leftarrow g_{1}^{\alpha_{g}}, \stackrel{\circ}{g}_{2} \leftarrow g_{2}^{\alpha_{g}}, \bar{g}_{1} \leftarrow g_{1}^{\bar{\alpha}}$, $\bar{g}_{1, \lambda_{1}} \leftarrow g_{1, \lambda_{1}}^{\bar{\alpha}}, \bar{g}_{2} \leftarrow g_{2}^{\bar{\alpha}}, \bar{g}_{2, \lambda_{1}} \leftarrow g_{2, \lambda_{1}}^{\bar{\alpha}}, \stackrel{\circ}{g}_{1, g / c} \leftarrow g_{1}^{\alpha_{g / c} \cdot\left(1-x^{\lambda_{1}}\right)}, \stackrel{\circ}{g}_{2, g / c} \leftarrow$ $g_{2}^{\alpha_{g / c} \cdot\left(1-x^{\lambda_{1}}\right)}, \stackrel{\circ}{g}_{1, f} \leftarrow g_{1}^{\alpha_{f}}, \stackrel{\circ}{g}_{2, f} \leftarrow g_{2}^{\alpha_{f}}, \stackrel{\circ}{g}_{1, h} \leftarrow g_{1}^{\alpha_{h}}$, and $\stackrel{\circ}{g}_{2, h} \leftarrow g_{2}^{\alpha_{h}}$. The common reference string is

$$
\begin{aligned}
\text { crs } \leftarrow & \left(\text { gk } ; g_{1}, g_{1, \lambda_{1}}, g_{2}, g_{2, \lambda_{1}}, \stackrel{\circ}{g}_{1}, \stackrel{\circ}{g}_{2}, \bar{g}_{1}, \bar{g}_{1, \lambda_{1}}, \bar{g}_{2}, \bar{g}_{2, \lambda_{1}}, \stackrel{\circ}{g}_{1, g / c}, \stackrel{\circ}{g}_{2, g / c}, \stackrel{\circ}{g}_{1, f}, \stackrel{\circ}{g}_{2, f},\right. \\
& \left.\stackrel{\circ}{1}_{1, h}, \stackrel{\circ}{g}_{2, h}\right) .
\end{aligned}
$$

A third party also creates $\mathrm{sk}:=\left(\mathrm{sk}_{1}, \mathrm{sk}_{2}\right) \leftarrow\left(\mathbb{Z}_{p}^{*}\right)^{2}$, and sets pk $:=$ $(f, h, \stackrel{\circ}{f}, \stackrel{\circ}{h}) \leftarrow\left(g_{1}^{1 / \mathrm{sk}_{1}}, g_{1}^{1 / \mathrm{sk}_{2}}, \stackrel{\circ}{g}_{1, f}^{1 / \mathrm{sk}_{1}}, \stackrel{\circ}{g}_{1, h}^{1 / \mathrm{sk}_{2}}\right)$.

Common inputs: (crs; pk, $\left.A_{g}, A_{f}, A_{h}, A_{c}\right)$, where pk $=(f, h, \stackrel{\circ}{f}, \stackrel{\circ}{h})$, $\left(A_{g}, A_{f}, A_{h}\right)=\left(g_{1}^{r_{f}+r_{h}+a}, f^{r_{f}}, h^{r_{h}}\right)$, and $A_{c}=g_{1}^{r_{f}+r_{h}} g_{1, \lambda_{1}}^{a}$.

Argument $\mathcal{P}\left(\mathrm{crs} ;\left(\mathrm{pk}, A_{g}, A_{f}, A_{h}, A_{c}\right),\left(a, r_{f}, r_{h}\right)\right)$ : let $\bar{A}_{c} \leftarrow \bar{g}_{1}^{r_{f}+r_{h}} \bar{g}_{1, \lambda_{1}}^{a}$, $\left(\stackrel{\circ}{A}_{g}, \stackrel{\circ}{A}_{f}, \stackrel{\circ}{A}_{h}\right) \leftarrow\left(\stackrel{\circ}{g}_{1}^{r_{f}+r_{h}+a}, \stackrel{\circ}{f}^{r_{f}}, \stackrel{\circ}{h}^{r_{h}}\right), \stackrel{\circ}{A}_{g / c} \leftarrow \stackrel{\circ}{g}_{1, g / c}^{a}$. Let $R_{f}, R_{h} \leftarrow \mathbb{Z}_{p}$. Let $\left(C_{f}, \bar{C}_{f}\right) \leftarrow\left(g_{2}^{R_{f}} g_{2, \lambda_{1}}^{r_{f}}, \bar{g}_{2}^{R_{f}} \bar{g}_{2, \lambda_{1}}^{r_{f}}\right),\left(C_{h}, \bar{C}_{h}\right) \leftarrow\left(g_{2}^{R_{h}} g_{2, \lambda_{1}}^{r_{h}}, \bar{g}_{2}^{R_{h}} \bar{g}_{2, \lambda_{1}}^{r_{h}}\right) \in \mathbb{G}_{2}^{2}$. Let $\left(\psi_{g}, \dot{\psi}_{g}\right) \leftarrow\left(g_{1}^{r+R_{f}+R_{h}}, \stackrel{\circ}{g}_{1}^{r+R_{f}+R_{h}}\right) \in \mathbb{G}_{1}^{2},\left(\psi_{f}, \dot{\psi}_{f}\right) \leftarrow\left(f^{R_{f}}, \stackrel{\circ}{f}^{R_{f}}\right) \in \mathbb{G}_{1}^{2}$, $\left(\psi_{h}, \dot{\circ}_{h}\right) \leftarrow\left(h^{R_{h}}, \stackrel{\circ}{h}^{R_{h}}\right) \in \mathbb{G}_{1}^{2}$.

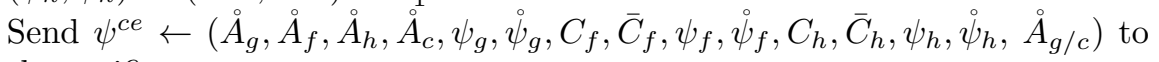
the verifier.

Verification $\mathcal{V}\left(\mathrm{crs} ;\left(\mathrm{pk}, A_{g}, A_{f}, A_{h}, A_{c}\right), \psi^{c e}\right)$ : Verify that $\hat{e}\left(\stackrel{\circ}{f}, g_{2}\right)=\hat{e}\left(f, \stackrel{\circ}{g}_{2, f}\right)$, $\hat{e}\left(\stackrel{\circ}{h}, g_{2}\right)=\hat{e}\left(h, \stackrel{\circ}{g}_{2, h}\right), \hat{e}\left(A_{g}, \stackrel{\circ}{g}_{2}\right)=\hat{e}\left(\stackrel{\circ}{A}_{g}, g_{2}\right), \hat{e}\left(A_{f}, \stackrel{\circ}{g}_{2, f}\right)=\hat{e}\left(\stackrel{\circ}{A}_{f}, g_{2}\right)$, $\hat{e}\left(A_{h}, \stackrel{\circ}{g}_{2, h}\right)=\hat{e}\left(\stackrel{\circ}{h}_{h}, g_{2}\right), \hat{e}\left(A_{c}, \bar{g}_{2}\right)=\hat{e}\left(\bar{A}_{c}, g_{2}\right), \hat{e}\left(\psi_{g}, \stackrel{\circ}{g}_{2}\right)=\hat{e}\left(\stackrel{\circ}{g}_{g}, g_{2}\right)$, $\hat{e}\left(\psi_{f}, \stackrel{\circ}{g}_{2, f}\right)=\hat{e}\left(\stackrel{\circ}{\psi}_{f}, g_{2}\right), \hat{e}\left(\psi_{h}, \stackrel{\circ}{g}_{2, h}\right)=\hat{e}\left(\stackrel{\circ}{\psi}_{h}, g_{2}\right), \hat{e}\left(\bar{g}_{1}, C_{f}\right)=\hat{e}\left(g_{1}, \bar{C}_{f}\right)$, $\hat{e}\left(\bar{g}_{1}, C_{h}\right)=\hat{e}\left(g_{1}, \bar{C}_{h}\right)$, and $\hat{e}\left(A_{g} / A_{c}, \stackrel{\circ}{g}_{2, g / c}\right)=\hat{e}\left(\AA_{g / c}, g_{2}\right)$.

Verify that $\hat{e}\left(f, C_{f}\right)=\hat{e}\left(\psi_{f}, g_{2}\right) \cdot \hat{e}\left(A_{f}, g_{2, \lambda_{1}}\right), \hat{e}\left(h, C_{h}\right)=\hat{e}\left(\psi_{h}, g_{2}\right)$. $\hat{e}\left(A_{h}, g_{2, \lambda_{1}}\right)$, and $\hat{e}\left(g_{1}, C_{f} C_{h}\right)=\hat{e}\left(\psi_{g} A_{c}^{-1}, g_{2}\right) \cdot \hat{e}\left(A_{g}, g_{2, \lambda_{1}}\right)$. 
As mentioned in Sect. 2, to prove the security of this argument, we will need a generalization of the PSDL and PKE assumptions.

Let $\Phi \subset \mathbb{Z}[X]$, with $d:=\max _{\varphi \in \Phi} \operatorname{deg} \varphi$, be a set of linearly independent polynomials, such that $|\Phi|$, all coefficients of all $\varphi \in \Phi$, and $d$ are polynomial in $\kappa$. Let 1 be the polynomial $\phi(x)=1$. We say that a bilinear group generator $\mathcal{G}_{\mathrm{bp}}$ is $\Phi$-PSDL secure, if for any non-uniform PPT adversary $\mathcal{A}$,

$$
\operatorname{Pr}\left[\begin{array}{l}
\mathrm{gk}:=\left(p, \mathbb{G}_{1}, \mathbb{G}_{2}, \mathbb{G}_{T}, \hat{e}\right) \leftarrow \mathcal{G}_{\mathrm{bp}}\left(1^{\kappa}\right), g_{1} \leftarrow \mathbb{G}_{1} \backslash\{1\}, g_{2} \leftarrow \mathbb{G}_{2} \backslash\{1\}, \\
x \leftarrow \mathbb{Z}_{p}: \mathcal{A}\left(\mathrm{gk} ;\left(g_{1}^{\varphi(x)}, g_{2}^{\varphi(x)}\right)_{\varphi \in\{1\} \cup \Phi}\right)=x
\end{array}\right]
$$

is negligible in $\kappa$. By a straightforward generalization of the proof from [15], any successful generic adversary for $\Phi$-PSDL requires time $\Omega(\sqrt{p / d})$, where $p$ is the group order.

Let $\Phi$ be as before. Consider $t \in\{1,2\}$. The bilinear group generator $\mathcal{G}_{\mathrm{bp}}$ is $\Phi$-PKE secure in group $\mathbb{G}_{t}$ if for any non-uniform PPT adversary $\mathcal{A}$ there exists a non-uniform PPT extractor $X_{\mathcal{A}}$,

$$
\operatorname{Pr}\left[\begin{array}{l}
\text { gk :=(p, } \left.\mathbb{G}_{1}, \mathbb{G}_{2}, \mathbb{G}_{T}, \hat{e}\right) \leftarrow \mathcal{G}_{\mathrm{bp}}\left(1^{\kappa}\right), g_{t} \leftarrow \mathbb{G}_{t} \backslash\{1\},(\hat{\alpha}, x) \leftarrow \mathbb{Z}_{p}^{2}, \\
\operatorname{crs} \leftarrow\left(\mathrm{gk} ;\left(g_{t}^{\varphi(x)}, g_{t}^{\hat{\alpha} \varphi(x)}\right)_{\varphi \in\{1\} \cup \Phi}\right),\left(c, \hat{c} ;\left(a_{\varphi}\right)_{\varphi \in\{0\} \cup \Phi}\right) \leftarrow\left(\mathcal{A} \| X_{\mathcal{A}}\right)(\mathrm{crs}): \\
\hat{c}=c^{\hat{\alpha}} \wedge c \neq \prod_{\varphi \in\{1\} \cup \Phi} g_{t}^{a_{\varphi} \varphi(x)}
\end{array}\right]
$$

is negligible in $\kappa$. Groth's proof [8] that the $[n]$-PKE assumption holds in the generic group model can be modified to the general case.

Note that $\mathcal{G}_{\mathrm{bp}}$ is $\Lambda$-PSDL secure (resp., $\Lambda$-PKE secure) iff it is $\left\{X^{\lambda}: \lambda \in \Lambda\right\}$ PSDL secure (resp., $\left\{X^{\lambda}: \lambda \in \Lambda\right\}$-PKE secure).

Theorem 1. The argument of this subsection is a perfectly argument for the next claim: for some $a, r_{f}, r_{h} \in \mathbb{Z}_{p}, A_{c}=g_{1}^{r} g_{1, \lambda_{1}}^{a}$ and $\left(A_{g}, A_{f}, A_{h}\right)=$ $\left(g^{r_{f}+r_{h}+a}, f^{r_{f}}, h^{r_{h}}\right)$. If the $\left\{1-X^{\lambda_{1}}\right\}-P S D L$ assumption and the $\left\{1-X^{\lambda_{1}}\right\}$ PKE assumption (in both $\mathbb{G}_{1}$ and $\mathbb{G}_{2}$ ) hold, then this argument is computationally sound. If the DLIN assumption holds in group $\mathbb{G}_{1}$, then this argument is computationally zero-knowledge.

(The proof of this theorem is given in App. A.) Clearly, this argument has CRS of length $\Theta(1)$, its argument consists of 13 elements of $\mathbb{G}_{1}$ and 2 elements of $\mathbb{G}_{2}$. The prover's computational complexity is dominated by 20 exponentiations. The verifier's computational complexity is dominated by 33 pairings.

\section{New Range Proof}

In the next range proof, the prover has an encrypted $a \in \mathbb{Z}_{p}$, and he aims to convince the verifier that $a \in[0, H]$. We will use the lifted BBS cryptosystem $\left(\mathcal{G}_{\mathrm{pkc}}, \mathcal{E} \mathrm{nc}, \mathcal{D}\right.$ ec $)$ that can be thought of as a perfectly binding commitment scheme if decryption is not necessary. Since we are interested in obtaining a sublinear 
argument, we will also use the (computationally binding) knowledge commitment scheme $\left(\mathcal{G}_{\text {com }}, \mathcal{C}\right.$ om $)$. We use the following result that was stated for $u=2$ in [16] and for general $u$ in [4].

Fact 6. Let $H>0$ and $u>1$. Let $\ell(u, H) \leq \log _{u}(H+1)$ be defined as in [4]. Then $a \in[0, H]$ if and only if for some $b_{i} \in[0, u-1]$,

$$
(u-1) a=\sum_{i=1}^{\ell(u,(u-1) H)} G_{i} b_{i}
$$

where $G_{i} \in \mathbb{Z}$ are values defined in [4]. That is, $(u-1) \cdot[0, H]=\sum_{i=1}^{\ell(u,(u-1) H)} G_{i}$. $[0, u-1]$. In particular, $[0, H]=\sum_{i=0}^{\left\lfloor\log _{2} H\right\rfloor}\left\lfloor\left(H+2^{i}\right) / 2^{i+1}\right\rfloor \cdot[0,1]$.

The precise values of $\ell(u, H)$ and $G_{i}$ are not important in the next description. It suffices to know that they can be efficiently evaluated. We note that

$$
G_{i}=\left\lfloor H / u^{i+1}\right\rfloor+\left\lfloor\left(H_{i}+\left(\sum_{j=0}^{i-1} H_{j} \bmod (u-1)\right)+1\right) / u\right\rfloor,
$$

where $H=\sum 2^{i} H_{i}$ [4].

The basic idea of the next range proof is as follows. Choose a $u>1$, and let $n=\ell(u,(u-1) H)$. According to Fact 6, $a \in[H]$ iff for $G_{i}$ computed as in Fact 6 , one has $(u-1) a=\sum_{i=1}^{n} G_{i} b_{i}$ for some $b_{i} \in[u-1]$. The prover shows by using a parallel version of range proof from [16 that for $i \in[n], b_{i} \in[0, u-1]$. The latter is done by writing $b_{i}$ as $b_{i}=\sum_{j=0}^{\left\lfloor\log _{2}(u-1)\right\rfloor} G_{j}^{\prime} b_{j i}^{\prime}$ (by again using Fact [6) and then showing that $b_{j i}^{\prime} \in[0,1]$ by using an Hadamard product arguments from [15. This will be achieved with commitments on $\left(b_{j 1}^{\prime}, \ldots, b_{j n}^{\prime}\right)$ for $j \in\left[\left\lfloor\log _{2}(u-1)\right\rfloor\right]$.

The prover then commits to the vector $\left(c_{1}, \ldots, c_{n}\right)$, where $c_{j}=\sum_{i=j}^{n} G_{i} b_{i}$, and shows that the values $c_{j}$ are correctly computed by using a small constant number of Hadamard product and permutation arguments. More precisely, he commits to $\left(G_{1} b_{1}, \ldots, G_{n} b_{n}\right)$ (and shows this has been done correctly), then to $\left(c_{2}, \ldots, c_{n}, c_{1}\right)$ (and shows this was done correctly), then to $\left(c_{2}, \ldots, c_{n}, 0\right)$ (and shows this was done correctly), and then shows that $\left(c_{1}, \ldots, c_{n}\right)=\left(G_{1} b_{1}, \ldots, G_{n} b_{n}\right)+\left(c_{2}, \ldots, c_{n}, 0\right)$. Thus, the verifier is convinced that $c_{j}=\sum_{i=j}^{n} G_{i} b_{i}$. Then, by Fact 6 , $c_{1}=$ $\sum_{i=1}^{n} G_{i} b_{i} \in(u-1) \cdot[H]$, and thus the prover has to show (by using a single product argument) that $\left(A_{c}^{u-1}, \hat{A}_{c}^{u-1}\right)$ commits to $\left(c_{1}, 0, \ldots, 0\right)$, and that $\left(A_{g}, A_{f}, A_{h}\right)$ is a lifted BBS encryption of $A$ with randomizer $\left(r_{f}, r_{h}\right)$ where $r=r_{f}+r_{h}$.

As in [15], in a few cases, instead of computing two different commitments $\mathcal{C o m}^{t}\left(\widehat{\mathrm{ck}}_{t} ; \boldsymbol{a} ; r\right)=\left(g_{t}^{r} \cdot \prod g_{t, \lambda_{i}}^{a_{i}}, \hat{g}_{t}^{r} \cdot \prod \hat{g}_{t \lambda_{i}}^{a_{i}}\right)$ and $\mathcal{C o m}^{t}\left(\widetilde{\mathrm{ck}}_{t} ; \boldsymbol{a} ; r\right)=\left(g_{t}^{r}\right.$. $\left.\prod g_{t, \lambda_{i}}^{a_{i}}, \tilde{g}_{t}^{r} \cdot \prod \tilde{g}_{t, \lambda_{i}}^{a_{i}}\right)$, we compute a composed commitment $\operatorname{Com}^{t}\left(\mathrm{ck}_{t} ; \boldsymbol{a} ; r\right)=$ $\left(g_{t}^{r} \cdot \prod g_{t, \lambda_{i}}^{a_{i}}, \hat{g}_{t}^{r} \prod \hat{g}_{t, \lambda_{i}}^{a_{i}}, \tilde{g}_{t}^{r} \cdot \prod \tilde{g}_{t, \lambda_{i}}^{a_{i}}\right)$.

The common input to both parties is equal to a BBS encryption $\left(A_{g}, A_{f}, A_{h}\right)$ of $a$, accompanied by $\left(A_{c}, \hat{A}_{c}\right)$ such that $\left(A_{c}, \hat{A}_{c}\right)$ is a knowledge commitment to $a$.

Theorem 2. Let $u>1$. Let $H=\operatorname{poly}(\kappa)$ and $n=\ell(u,(u-1) H)$ where $\ell$ is defined as in Fact [6. Let $\Lambda=\left\{\lambda_{i}\right\}_{i \in[n]}$ be an $(n, \kappa)$-nice tuple. Denote $\lambda_{0}:=0$. 
System parameters: $H, G_{i}, n, u, n_{v}:=\left\lfloor\log _{2}(u-1)\right\rfloor$, and $G_{j}^{\prime}:=\left\lfloor\left(u+2^{j}\right) / 2^{j+1}\right\rfloor$.

Common reference string generation $\mathcal{G}_{\mathrm{crs}}\left(1^{\kappa}\right)$ : Set gk := $\left(p, \mathbb{G}_{1}, \mathbb{G}_{2}, \mathbb{G}_{T}, \hat{e}\right) \leftarrow \mathcal{G}_{\mathrm{bp}}\left(1^{\kappa}\right)$. Generate random $\widehat{\alpha}, \tilde{\alpha}, \alpha_{g}, \alpha_{f}, \alpha_{h}, \bar{\alpha}, \alpha_{g / c}, x \leftarrow \mathbb{Z}_{p}$. Let $g_{1} \leftarrow \mathbb{G}_{1} \backslash\{1\}$ and $g_{2} \leftarrow \mathbb{G}_{2} \backslash\{1\}$. Denote $g_{t s} \leftarrow g_{t}^{x^{s}}, \hat{g}_{t s} \leftarrow g_{t}^{\widehat{\alpha} x^{s}}, \tilde{g}_{t s} \leftarrow g_{t}^{\tilde{\alpha} x^{s}}, \stackrel{\circ}{g}_{1} \leftarrow g_{1}^{\alpha_{g}}, \stackrel{\circ}{g}_{2} \leftarrow g_{2}^{\alpha_{g}}, \overline{g_{1}} \leftarrow g_{1}^{\bar{\alpha}}$, $\bar{g}_{1, \lambda_{1}} \leftarrow g_{1, \lambda_{1}}^{\bar{\alpha}}, \bar{g}_{2} \leftarrow g_{2}^{\bar{\alpha}}, \bar{g}_{2, \lambda_{1}} \leftarrow g_{2, \lambda_{1}}^{\bar{\alpha}}, \stackrel{\circ}{g}_{1, g / c} \leftarrow g_{1}^{\alpha_{g / c} \cdot\left(1-x^{\lambda_{1}}\right)}, \stackrel{\circ}{g}_{2, g / c} \leftarrow g_{2}^{\alpha_{g / c} \cdot\left(1-x^{\lambda_{1}}\right)}$, $\stackrel{\circ}{g}_{1, f} \leftarrow g_{1}^{\alpha_{f}}, \stackrel{\circ}{g}_{2, f} \leftarrow g_{2}^{\alpha_{f}}, \stackrel{\circ}{g}_{1, h} \leftarrow g_{1}^{\alpha_{h}}$, and $\stackrel{\circ}{g}_{2, h} \leftarrow g_{2}^{\alpha_{h}}$. Set $D \leftarrow \prod_{=1}^{n} g_{1, \lambda_{i}}$, $E_{\mathrm{rot}} \leftarrow \prod_{i=1}^{n} g_{2,2 \lambda_{\mathrm{rot}(i)}-\lambda_{i}}$, and $\widetilde{E}_{\mathrm{rot}} \leftarrow E_{\mathrm{rot}}^{\tilde{\alpha}}$. The common reference string is crs $\leftarrow$ (gk; $\left.\left(g_{1, s}, \hat{g}_{1, s}, \tilde{g}_{1, s}\right)_{s \in\{0\} \cup \Lambda}, g_{2},\left(\hat{g}_{2, s}\right)_{s \in \hat{\Lambda}},\left(g_{2, s}, \tilde{g}_{2, s}\right)_{s \in \tilde{\Lambda}}, D, E_{\mathrm{rot}}, \widetilde{E}_{\mathrm{rot}}\right)$.

Set $\mathrm{ck}_{1} \leftarrow\left(\mathrm{gk} ;\left(g_{1 s}, \hat{g}_{1 s}, \tilde{g}_{1 s}\right)_{s \in\{0\} \cup \Lambda}\right), \widehat{c k}_{1} \leftarrow\left(\mathrm{gk} ;\left(g_{1 s}, \hat{g}_{1 s}\right)_{s \in\{0\} \cup \Lambda}\right)$ and $\widetilde{c k}_{1} \leftarrow$ $\left(\mathrm{gk} ;\left(g_{1 s}, \tilde{g}_{1 s}\right)_{s \in\{0\} \cup \Lambda}\right)$. The prover creates a secret key sk $:=\left(\mathrm{sk}_{1}, \mathrm{sk}_{2}\right) \leftarrow \mathbb{Z}_{p}^{2}$, and sets pk $\leftarrow(f, h, \stackrel{f}{f}, h) \leftarrow\left(g_{1}^{1 / \mathrm{sk}_{1}}, g_{1}^{1 / \mathrm{sk}_{2}} \stackrel{\circ}{g}_{1, f}^{1 / \mathrm{sk}_{1}}, \stackrel{\circ}{g}_{1, h}^{1 / \mathrm{sk}_{2}}\right)$. Here, $\mathcal{E} \mathrm{nc}_{\mathrm{pk}}\left(m ;\left(r_{f}, r_{h}\right)\right):=$ $\left(g_{1}^{r_{f}+r_{h}+m}, f^{r_{f}}, h^{r_{h}}\right)$.

Common inputs: (pk, $\left.A_{g}, A_{f}, A_{h}, A_{c}, \hat{A}_{c}\right)$, where $\left(A_{g}, A_{f}, A_{h}\right)=\left(g_{1}^{r+a}, f^{r_{f}}, h^{r_{h}}\right)$ and $\left.\left(A_{c}, \hat{A}_{c}\right)=g_{1}^{r} g_{1, \lambda_{1}}^{a}, \hat{g}_{1}^{r} \hat{g}_{1, \lambda_{1}}^{a}\right)$, for $r=r_{f}+r_{h}$.

Argument $\mathcal{P}\left(\mathrm{crs} ;\left(\mathrm{pk}, A_{g}, A_{f}, A_{h}, A_{c}, \hat{A}_{c}\right),\left(a, r_{f}, r_{h}\right)\right)$ : The prover does the following:

1. Compute $\left(b_{1}, \ldots, b_{n}\right) \in \mathbb{Z}_{u}^{n}$ such that $(u-1) a=\sum_{i=1}^{n} G_{i} b_{i}$.

2. For $i \in[n]$ do: compute $\left(b_{0 i}^{\prime}, \ldots, b_{n_{v}, i}^{\prime}\right) \in \mathbb{Z}_{2}^{n_{v}+1}$ such that $b_{i}=\sum_{j=0}^{n_{v}} G_{j}^{\prime} \cdot b_{j i}^{\prime}$.

3. For $j \in\left[0, n_{v}\right]$ do:

- Let $r_{j} \leftarrow \mathbb{Z}_{p},\left(B_{j}^{\prime}, \hat{B}_{j}^{\prime}\right) \leftarrow \mathcal{C o m}^{1}\left(\widehat{\mathrm{ck}}_{1} ; b_{j 1}^{\prime}, \ldots, b_{j n}^{\prime} ; r_{j}\right), B_{j 2}^{\prime} \leftarrow g_{2}^{r_{j}} \cdot \prod_{i=1}^{n} g_{2, \lambda_{i}}^{b_{j i}^{\prime}}$.

- Create an argument $\left(\psi_{j}^{\prime}, \hat{\psi}_{j}^{\prime}\right)$ for $\llbracket\left(B_{j}^{\prime}, \hat{B}_{j}^{\prime}\right) \rrbracket \circ \llbracket\left(B_{j}^{\prime}, \hat{B}_{j}^{\prime}, B_{j 2}^{\prime}\right) \rrbracket=\llbracket\left(B_{j}^{\prime}, \hat{B}_{j}^{\prime}\right) \rrbracket$.

4. For $i \in[n]$, let $c_{i} \leftarrow \sum_{k=i}^{n} G_{k} b_{k}$.

5. Set $r_{0}^{\prime}, r_{1}^{\prime}, r_{2}^{\prime} \leftarrow \mathbb{Z}_{p},\left(B^{\dagger}, \hat{B}^{\dagger}\right) \leftarrow \mathcal{C o m}^{1}\left(\widehat{c k}_{1} ; G_{1} b_{1}, \ldots, G_{n} b_{n} ; r_{0}^{\prime}\right),(C, \hat{C}, \tilde{C}) \leftarrow$ $\operatorname{Com}^{1}\left(\mathrm{ck}_{1} ; \boldsymbol{c} ; r_{1}^{\prime}\right)$, and $\left(C_{\text {rot }}, \hat{C}_{\text {rot }}, \tilde{C}_{\text {rot }}\right) \leftarrow \operatorname{Com}^{1}\left(\mathrm{ck}_{1} ; c_{2}, \ldots, c_{n-1}, c_{n}, c_{1} ; r_{2}^{\prime}\right)$.

6. Create an argument $\left(\psi_{1}^{\times}, \hat{\psi}_{1}^{\times}\right)$for $\llbracket\left(\prod_{j=0}^{n_{v}}\left(B_{j}^{\prime}\right)^{G_{j}^{\prime}}, \prod_{j=0}^{n_{v}}\left(\hat{B}_{j}^{\prime}\right)^{G_{j}^{\prime}}\right) \rrbracket \quad \circ$ $\llbracket\left(\mathcal{C o m}^{1}\left(\widehat{\mathrm{ck}}_{1} ; G_{1}, \ldots, G_{n} ; 0\right), \prod_{i=1}^{n} g_{2, \lambda_{i}}^{G_{i}}\right) \rrbracket=\llbracket\left(B^{\dagger}, \hat{B}^{\dagger}\right) \rrbracket$.

7. Create an argument $\left(A^{*}, \hat{A}^{*}, \psi_{2}^{\times}, \hat{\psi}_{2}^{\times}, \psi_{2}^{\text {rot }}, \hat{\psi}_{2}^{\text {rot }}\right) \quad$ for $\quad \operatorname{rot}(\llbracket(C, \tilde{C}) \rrbracket)=$ $\llbracket\left(C_{\text {rot }}, \hat{C}_{\text {rot }}, \tilde{C}_{\text {rot }}\right) \rrbracket$.

8. Create an argument $\left(\psi_{3}^{\times}, \hat{\psi}_{3}^{\times}\right)$for $\llbracket\left(C_{\text {rot }}, \hat{C}_{\text {rot }}\right) \rrbracket$ ○ $\llbracket\left(\operatorname{Com}^{1}\left(\widehat{c k}_{1} ; 1,1, \ldots, 1,0 ; 0\right), \prod_{i=1}^{n-1} g_{2, \lambda_{i}}\right) \rrbracket=\llbracket\left(C / B^{\dagger}, \hat{C} / \hat{B}^{\dagger}\right) \rrbracket$.

9. Create an argument $\left(\psi_{4}^{\times}, \hat{\psi}_{4}^{\times}\right)$for $\llbracket(C, \hat{C}) \rrbracket \circ \llbracket\left(\operatorname{Com}^{1}\left(\widehat{c k}_{1} ; 1,0, \ldots, 0,0 ; 0\right), g_{2, \lambda_{1}}\right) \rrbracket=$ $\llbracket\left(A_{c}^{u-1}, \hat{A}_{c}^{u-1}\right) \rrbracket$.

10. Create an argument $\psi_{5}^{\text {ce }}$ that $A_{c}$ commits to the same value that $\left(A_{g}, A_{f}, A_{h}\right)$ encrypts.

11. Send $\psi \leftarrow \quad\left(\left(B_{j}^{\prime}, \hat{B}_{j}^{\prime}, B_{j 2}^{\prime}, \psi_{j}^{\prime}, \hat{\psi}_{j}^{\prime}\right)_{j \in\left[0, n_{v}\right]},\left(B^{\dagger}, \hat{B}^{\dagger}\right),(C, \hat{C}, \tilde{C}),\left(C_{\text {rot }}, \hat{C}_{\text {rot }}, \tilde{C}_{\text {rot }}\right)\right.$, $\left.\left(\psi_{1}^{\times}, \hat{\psi}_{1}^{\times}\right),\left(A^{*}, \hat{A}^{*}, \psi_{2}^{\times}, \hat{\psi}_{2}^{\times}, \psi_{2}^{\text {rot }}, \hat{\psi}_{2}^{\text {rot }}\right),\left(\psi_{3}^{\times}, \hat{\psi}_{3}^{\times}\right),\left(\psi_{4}^{\times}, \hat{\psi}_{4}^{\times}\right), \psi_{5}^{c e}\right)$ to $\mathcal{V}$.

Verification $\mathcal{V}\left(\mathrm{crs} ;\left(\mathrm{pk}, A_{g}, A_{f}, A_{h}, A_{c}, \hat{A}_{c}\right), \psi\right): \mathcal{V}$ does the following.

1. For $j \in\left[0, n_{v}\right]$ do:

(a) Check that $\hat{e}\left(B_{j}^{\prime}, g_{2}\right)=\hat{e}\left(g_{1}, B_{j 2}^{\prime}\right)$ and $\hat{e}\left(B_{j}^{\prime}, \hat{g}_{2}\right)=\hat{e}\left(\hat{B}_{j}^{\prime}, g_{2}\right)$.

(b) Verify $\left(\psi_{j}^{\prime}, \hat{\psi}_{j}^{\prime}\right)$ for inputs as specified above.

2. For $K \in\left\{A_{c}, B^{\dagger}, C, C_{\text {rot }}\right\}$ : check that $\hat{e}\left(K, \hat{g}_{2}\right)=\hat{e}\left(\hat{K}, g_{2}\right)$.

3. For $K \in\left\{C, C_{\text {rot }}\right\}$ : check that $\hat{e}\left(K, \tilde{g}_{2}\right)=\hat{e}\left(\tilde{K}, g_{2}\right)$.

4. Verify the arguments $\left(\psi_{1}^{\times}, \hat{\psi}_{1}^{\times}\right),\left(A^{*}, \hat{A}^{*}, \psi_{2}^{\times}, \hat{\psi}_{2}^{\times}, \psi_{2}^{\text {rot }}, \hat{\psi}_{2}^{\text {rot }}\right),\left(\psi_{3}^{\times}, \hat{\psi}_{3}^{\times}\right)$, $\left(\psi_{4}^{\times}, \hat{\psi}_{4}^{\times}\right), \psi_{5}^{\text {ce }}$ for inputs as specified above.

Protocol 3. The new range proof 
Let $\widehat{\Lambda}:=\{0\} \cup \Lambda \cup 2^{\widehat{ }} \Lambda$, and $\tilde{\Lambda}$ be as in Sect. 3.2. Let rot $\in S_{n}$ be such that $\operatorname{rot}(i)=i-1$ if $i>1$, and $\operatorname{rot}(1)=n$. Define $G_{i}$ as in Fact [6. The argument in Prot. 3 is perfectly complete. If $\mathcal{G}_{\mathrm{bp}}$ is $\left\{1-X^{\lambda_{1}}\right\}-P K E, \Lambda-P K E$ and DLIN secure in $\mathbb{G}_{1}$, then the argument in Prot. 3 is computationally zero-knowledge. If $\mathcal{G}_{\mathrm{bp}}$ is $\left(\left\{X^{s}\right\}_{s \in \tilde{\Lambda}} \cup\left\{1-X^{\lambda_{1}}\right\}\right)-P S D L, \Lambda$-PKE and $\left\{1-X^{\lambda_{1}}\right\}$-PKE secure in both $\mathbb{G}_{1}$ and $\mathbb{G}_{2}$, then the argument in Prot. 3 is computationally sound.

This argument is computationally zero-knowledge because $\left(A_{c}, \hat{A}_{c}\right)$ was provided by a prover and not generated during the argument. To achieve perfect zeroknowledge, one must be able to open $\left(A_{c}, \hat{A}_{c}\right)$ given only the CRS trapdoor. That is, one has to use an extractable commitment scheme. It is easy to see that the knowledge commitment scheme is extractable, however, extractability is only achieved under the PKE assumption. The use of a cryptosystem also makes achieving perfect zero-knowledge impossible.

Proof ([Of Thm. Q2). Perfect Completeness: Recall that in the case of the product arguments, the inputs of $\mathcal{P}$ are $\left(A, \hat{A}, B, \hat{B}, B_{2}, C, \hat{C}\right)$. Within this proof we say that $\left(B, \hat{B}, B_{2}\right)$ (assuming $B_{2}$ is correctly defined, that is, $\hat{e}\left(B, g_{2}\right)=$ $\left.\hat{e}\left(g_{1}, B_{2}\right)\right)$ commits to the same values as $(B, \hat{B})$.

The pairing verifications (for example, that $\left.\hat{e}\left(K, \hat{g}_{2}\right)=\hat{e}\left(\hat{K}, g_{2}\right)\right)$ hold by construction of the protocol. Since $\left(B_{j}^{\prime}, \hat{B}_{j}^{\prime}\right)$ commits to $\left(b_{j 1}^{\prime}, \ldots, b_{j n}^{\prime}\right)$ for binary $b_{j i}^{\prime}$ then the argument $\left(\psi_{j}^{\prime}, \hat{\psi}_{j}^{\prime}\right)$ verifies.

Note that $\left(\prod_{j=0}^{n_{v}}\left(B_{j}^{\prime}\right)^{G_{j}^{\prime}}, \prod_{j=0}^{n_{v}}\left(\hat{B}_{j}^{\prime}\right)^{G_{j}^{\prime}}\right)$ commits to $\left(b_{1}, \ldots, b_{n}\right)$. Thus argument $\left(\psi_{1}^{\times}, \hat{\psi}_{1}^{\times}\right)$verifies. Since $\left(C_{\text {rot }}, \hat{C}_{\text {rot }}\right)$ commits to a rotation of $(C, \hat{C})$, then $\left(A^{*}, \hat{A}^{*}, \psi_{2}^{\times}, \hat{\psi}_{2}^{\times}, \psi_{2}^{\text {rot }}, \hat{\psi}_{2}^{\text {rot }}\right)$ verifies. Since $\left(C_{\text {rot }}, \hat{C}_{\text {rot }}\right)$ commits to $\left(0, c_{1}, \ldots, c_{n-1}\right)$ and $\left(C / B^{\dagger}, \hat{C} / \hat{B}^{\dagger}\right)$ commits to $\left(c_{1}-G_{1} b_{1}, c_{2}-G_{2} b_{2}, \ldots, c_{n}-G_{n} b_{n}\right)=$ $\left(0, c_{1}, \ldots, c_{n-1}\right)$, then $\left(\psi_{3}^{\times}, \hat{\psi}_{3}^{\times}\right)$verifies. Finally, since $(u-1) a=\sum_{i=1}^{n} G_{i} b_{i}$ and $c_{n}=\sum_{i=1}^{n} G_{i} b_{i}$, then $\left(\psi_{4}^{\times}, \hat{\psi}_{4}^{\times}\right)$verifies.

Computational soundness: let $\mathcal{A}$ be a non-uniform PPT adversary who creates a statement (pk, $\left.A_{g}, A_{f}, A_{h}, A_{c}, \hat{A}_{c}\right)$ and an accepting range proof $\psi$. By the DLIN assumption, the BBS cryptosystem is IND-CPA secure, and thus the adversary obtains no information from $\left(A_{g}, A_{f}, A_{h}\right)$. By the $\Lambda$-PKE and the $\left\{1-X^{\lambda_{1}}\right\}$-PKE assumptions, there exists a non-uniform PPT extractor $X_{\mathcal{A}}$ that, running on the same inputs and seeing $\mathcal{A}$ 's random tape, extracts the following openings:

$-\left(A_{c}, \hat{A}_{c}\right)=\mathcal{C o m}^{1}\left(\widehat{c k}_{1} ; \boldsymbol{a} ; r\right),\left(B_{j}^{\prime}, \hat{B}_{j}^{\prime}\right)=\mathcal{C o m}^{1}\left(\widehat{c k}_{1} ; \boldsymbol{b}_{j}^{\prime} ; r_{j}\right)$ for $j \in\left[0, n_{v}\right]$,

$-\left(B^{\dagger}, \hat{B}^{\dagger}\right)=\mathcal{C o m}^{1}\left(\widehat{c k}_{1} ; \boldsymbol{b}^{\dagger} ; r_{0}^{\prime}\right)$,

- $(C, \hat{C})=\mathcal{C o m}^{1}\left(\widehat{c k}_{1} ; c ; r_{1}^{\prime}\right)$ and $\left(C_{\text {rot }}, \hat{C}_{\text {rot }}\right)=\mathcal{C o m}^{1}\left(\widehat{c k}_{1} ; c_{\text {rot }} ; r_{2}^{\prime}\right)$,

$-\left(\psi_{1}^{\times}, \hat{\psi}_{1}^{\times}\right)=\left(\prod_{s \in \hat{\Lambda}} g_{2 s}^{f_{(\times 1, s)}^{\prime}}, \prod_{s \in \hat{\Lambda}} \hat{g}_{2 s}^{f_{(\times 1, s)}^{\prime}}\right)$,

- $\left(A^{*}, \hat{A}^{*}\right)=\mathcal{C o m}^{1}\left(\widehat{c k}_{1} ; \boldsymbol{a}^{*} ; r_{a^{*}}\right)$,

$-\left(\psi_{2}^{\times}, \hat{\psi}_{2}^{\times}\right)=\left(\prod_{s \in \hat{\Lambda}} g_{2 s}^{f_{(\times 2, s)}^{\prime}}, \prod_{s \in \hat{\Lambda}} \hat{g}_{2 s}^{\left.f_{(\times 2, s)}^{\prime}\right)}\right)$,

$-\left(\psi_{2}^{\text {rot }}, \hat{\psi}_{2}^{\text {rot }}\right)=\left(\prod_{s \in \tilde{\Lambda}} g_{2 s}^{f_{(\mathrm{rot} 2, s)}^{\prime}}, \prod_{s \in \tilde{\Lambda}} \tilde{g}_{2 s}^{f_{(\mathrm{rot} 2, s)}^{\prime}}\right)$, 


$$
\begin{aligned}
& -\left(\psi_{3}^{\times}, \hat{\psi}_{3}^{\times}\right)=\left(\prod_{s \in \hat{\Lambda}} g_{2 s}^{\left.f_{(\times 3, s)}^{\prime}, \prod_{s \in \hat{\Lambda}} \hat{g}_{2 s}^{f_{(\times 3, s)}^{\prime}}\right), \text { and }}\right. \\
& -\left(\psi_{4}^{\times}, \hat{\psi}_{4}^{\times}\right)=\left(\prod_{s \in \hat{\Lambda}} g_{2 s}^{f_{(\times 4, s)}^{\prime}}, \prod_{s \in \hat{\Lambda}} \hat{g}_{2 s}^{f_{(\times 4, s)}^{\prime}}\right) .
\end{aligned}
$$

It will also create the openings that correspond to $\psi_{5}^{c e}$. If any of the openings fails, we are done. Since $\tilde{\Lambda}$-PSDL and $\left\{1-X^{\lambda_{1}}\right\}$-PSDL assumptions are supposed to hold, all the following is true. (If it is not true, one can efficiently test it, and thus we have broken the PSDL assumption.)

Since $\hat{e}\left(B_{j}^{\prime}, g_{2}\right)=\hat{e}\left(g_{1}, B_{j 2}^{\prime}\right)$ for $j \in\left[0, n_{v}\right]$, then $\left(B_{j 1}, \hat{B}_{j 1}, B_{j 2}\right)$ commits to $\boldsymbol{b}_{\boldsymbol{j}}^{\prime}$. Therefore, due to the $\hat{\Lambda}$-PSDL assumption, the fact that the adversary knows the openings of $\left(B_{j}^{\prime}, \hat{B}_{j}^{\prime}\right)$ and $\left(\psi_{j}^{\prime}, \hat{\psi}_{j}^{\prime}\right)$, and the last statement of Fact 2. since $\left(\psi_{j}^{\prime}, \hat{\psi}_{j}^{\prime}\right)$ verifies, then $b_{j i}^{\prime} \in\{0,1\}$ for all $j \in\left[0, n_{v}\right]$ and $i \in[1, n]$. Thus, by Fact 6. $\boldsymbol{b}=\left(b_{1}, \ldots, b_{n}\right):=\left(\sum_{j=0}^{n_{v}} G_{j}^{\prime} b_{j 1}^{\prime}, \ldots, \sum_{j=0}^{n_{v}} G_{j}^{\prime} b_{j n}^{\prime}\right) \in[0, u-1]^{n}$, and thus $\left(\prod_{j=0}^{n_{v}}\left(B_{j}^{\prime}\right)^{G_{j}^{\prime}}, \prod_{j=0}^{n_{v}}\left(\hat{B}_{j}^{\prime}\right)^{G_{j}^{\prime}}\right)$ commits to $\boldsymbol{b}$ with $b_{i} \in[0, u-1]$.

Due to the $\hat{\Lambda}$-PSDL assumption, the fact that the adversary knows the openings of $\left(B_{j}^{\prime}, \hat{B}_{j}^{\prime}\right),\left(B^{\dagger}, \hat{B}^{\dagger}\right)$ and $\left(\psi_{1}^{\times}, \hat{\psi}_{1}^{\times}\right)$, and the last statement of Fact 2, since $\left(\psi_{1}^{\times}, \hat{\psi}_{1}^{\times}\right)$verifies, then $b_{i}^{\dagger}=G_{i} b_{i}$. Due to the $\tilde{\Lambda}$-PSDL assumption, the fact that the adversary knows the openings of $(C, \tilde{C}),\left(C_{\text {rot }}, \hat{C}_{\text {rot }}\right)$ and

$$
\left(A^{*}, \hat{A}^{*}, \psi_{2}^{\times}, \hat{\psi}_{2}^{\times}, \psi_{2}^{\text {rot }}, \hat{\psi}_{2}^{\text {rot }}\right)
$$

and the last statement of Fact 2 since $\left(A^{*}, \hat{A}^{*}, \psi_{2}^{\times}, \hat{\psi}_{2}^{\times}, \psi_{2}^{\text {rot }}, \hat{\psi}_{2}^{\text {rot }}\right)$ verifies, then $c_{\text {rot }, 1}=c_{n}$ and $c_{\text {rot }, i+1}=c_{i}$ for $i \geq 1$.

Due to the $\hat{\Lambda}$-PSDL assumption, the fact that the adversary knows the openings of $\left(C_{\text {rot }}, \tilde{C}_{\text {rot }}\right),(C, \hat{C}),\left(B^{\dagger}, \hat{B}^{\dagger}\right)$, and $\left(\psi_{3}^{\times}, \hat{\psi}_{3}^{\times}\right)$, and the last statement of Fact 2, since $\left(\psi_{3}^{\times}, \hat{\psi}_{3}^{\times}\right)$verifies, then $c_{1}-G_{1} b_{1}=0$ and $c_{i}-G_{i} b_{i}=c_{\text {rot }, i}=c_{i-1}$ for $i>1$. Therefore, $c_{1}=G_{1} b_{1}, c_{2}=G_{2} b_{2}+G_{1} b_{1}$, and by induction $c_{i}=\sum_{j=1}^{n} G_{i} b_{i}$ for $i \geq 1$. In particular, $c_{n}=\sum_{i=1}^{n} G_{i} b_{i}$ for $b_{i} \in[0, u-1]$.

Due to the $\hat{\Lambda}$-PSDL assumption, the fact that the adversary knows the openings of $(C, \hat{C}),\left(A_{c}, \hat{A}_{c}\right)$, and $\left(\psi_{4}^{\times}, \hat{\psi}_{4}^{\times}\right)$, and the last statement of Fact 2] since $\left(\psi_{4}^{\times}, \hat{\psi}_{4}^{\times}\right)$verifies, then $\left(A_{c}, \hat{A}_{c}\right)=\left(g_{1}^{r} g_{1, \lambda_{1}}^{a}, \hat{g}_{1}^{r} \hat{g}_{1, \lambda_{1}}^{a}\right)$ commits to $(a, 0, \ldots, 0)$ such that $(u-1) a=\sum_{i=1}^{n} G_{i} b_{i}$ for $b_{i} \in[0, u-1]$, and therefore by Fact 6 , $a \in[0, H]$.

Due to the $\left\{1-X^{\lambda_{1}}\right\}$-PSDL assumption and since $\psi_{5}^{c e}$ verifies, then $\left(A_{g}, A_{f}, A_{h}\right)$ encrypts $a \in[0, H]$.

COMPUTATIONAL ZERO-KNOWLEDGE: we construct the following simulator $\mathcal{S}=\left(\mathcal{S}_{1}, \mathcal{S}_{2}\right)$. First, $\mathcal{S}_{1}$ creates a correctly formed common reference string together with a simulation trapdoor $\mathrm{td}=(\hat{\alpha}, \tilde{\alpha}, \ldots, x)$. After that, the prover creates a statement $i n p^{r}:=\left(\mathrm{pk}, A_{g}, A_{f}, A_{h}, A_{c}, \hat{A}_{c}\right)$ and sends it to the simulator. Second, $\mathcal{S}_{2}\left(\mathrm{crs} ; i n p^{r} ; \mathrm{td}\right)$ uses a knowledge extractor to extract $(\boldsymbol{a}, r)$ from the prover's random coins and $\left(A_{c}, \hat{A}_{c}\right)$. Since we are only interested in the case of a honest prover, we have that $\boldsymbol{a}=(a, 0, \ldots, 0)$ with $a \in[0, H]$. Thus, using the fact that the knowledge commitment scheme is also trapdoor, the simulator computes $r^{\prime \prime} \leftarrow a x^{\lambda_{n}}+r$; clearly $A=g_{1}^{r^{\prime \prime}}$. Since both $r$ and $r^{\prime \prime}$ are uniformly random, $r^{\prime \prime}$ does not leak any information on the prover's input. After that, the simulator creates all commitments $\left(B_{j}^{\prime}, \hat{B}_{j}^{\prime}, B_{j 2}^{\prime}\right)_{j \in\left[0, n_{v}\right]},\left(B^{\dagger}, \hat{B}^{\dagger}\right),(C, \hat{C}, \tilde{C})$ and 
$\left(C_{\text {rot }}, \hat{C}_{\text {rot }}, \tilde{C}_{\text {rot }}\right)$ as in the argument, but replacing $\boldsymbol{a}$ with 0 and $r$ with $r^{\prime \prime}$. (Note that all the mentioned commitments just commit to $\mathbf{0}$.) Thus, the simulator can simulate all product and permutation arguments and the argument of Sect. 5 . Clearly, this simulated argument $\psi^{\text {sim }}$ is perfectly indistinguishable from the real argument $\psi$.

Theorem 3. Let $u>1$. Let $\Lambda$ be as in Fact 1 and let $n=\ell(u,(u-1) H) \leq$ $\left\lfloor\log _{u}((u-1) H+1)\right\rfloor \approx \log H / \log u+1$, where $\ell(\cdot, \cdot)$ is defined as in Fact $[6$. Let $n_{v}=\left\lceil\log _{2}(u-1)\right\rceil$. Assume that we use the Hadamard product argument and the permutation argument from Sect. 3 . The range proof in Prot. 3 has a length- $n^{1+o(1)}$ common reference string, communication of $2 n_{v}+25$ elements from $\mathbb{G}_{1}$ and $3 n_{v}+15$ elements from $\mathbb{G}_{2}$, the prover's computational complexity of $\Theta\left(n^{2} n_{v}\right)$ scalar multiplications in $\mathbb{Z}_{p}$ and $n^{1+o(1)} n_{v}$ exponentiations in $\mathbb{G}_{1}$ or $\mathbb{G}_{2}$. The verifier's computational complexity is dominated by $9 n_{v}+81$ pairings.

Proof. The communication complexity: $n_{v}+1$ tuples $\left(B_{j}^{\prime}, \hat{B}_{j}^{\prime}, B_{j 2}^{\prime}, \psi_{j}\right)$ (each has 2 elements of $\mathbb{G}_{1}$ and 3 elements of $\mathbb{G}_{2}$ ), and then 8 extra elements from $\mathbb{G}_{1}$, 3 Hadamard product arguments (2 elements from $\mathbb{G}_{2}$ each), 1 permutation argument ( 2 elements from $\mathbb{G}_{1}$ and 4 elements from $\left.\mathbb{G}_{2}\right)$, and argument $\psi^{\text {ce }}(13$ elements from $\mathbb{G}_{1}$ and 2 elements from $\left.\mathbb{G}_{2}\right)$. In total, thus $2\left(n_{v}+1\right)+8+2+13=$ $2 n_{v}+25$ elements from $\mathbb{G}_{1}$ and $3\left(n_{v}+1\right)+3 \cdot 2+4+2=3 n_{v}+15$ elements from $\mathbb{G}_{2}$.

The prover's computational complexity is dominated by $\left(n_{v}+1\right)+3=n_{v}+4$ Hadamard product arguments and 1 permutation argument $\left(\Theta\left(n^{2}\right)\right.$ scalar multiplications and bilinear-group $n^{1+o(1)}$ exponentiations each), that is in total $\Theta\left(n^{2} \cdot n_{v}\right)=\Theta\left(n^{2} \cdot \log u\right)$ scalar multiplications and $n^{1+o(1)} \log u$ exponentiations.

The verifier's computational complexity is dominated by verifying $n_{v}+4$ Hadamard product arguments (5 pairings each), 1 permutation argument (12 pairings), and the argument $\psi^{c e}$ (33 pairings). In addition, the verifier performs $2 \cdot\left(2\left(n_{v}+1\right)+6\right)=4 n_{v}+16$ pairings. The total number of pairings is thus $9 n_{v}+81$. The rest follows.

The communication complexity is minimized when $n_{v}$ (and thus $u$ ) is as small as possible, that is, $u=2$. Then $n_{v}=\left\lfloor\log _{2} 1\right\rfloor=0$. In this case the communication consists of 12 elements from $\mathbb{G}_{1}$ and 13 elements from $\mathbb{G}_{2}$. The same choice $u=2$ is also optimal for verifier's computational complexity (81 pairings). As noted before, at the security level of $2^{128}$, elements of $\mathbb{G}_{1}$ can be represented in 256 bits, and elements of $\mathbb{G}_{2}$ in 512 bits. Thus, at this security level, if $u=2$ then the communication is $25 \cdot 256+25 \cdot 512=14080 \mathrm{bits}$, that is, only about 4 to 5 times longer than the current recommended length of a $2^{128}$-secure RSA modulus. Therefore, the communication of the new range proof is even smaller than that of Lagrange theorem based arguments like [13].

The optimal prover's computational complexity is achieved when the number of exponentiations, $n^{1+o(1)} \cdot n_{v}=(\log H / \log u)^{1+o(1)} \cdot\left\lfloor\log _{2}(u-1)\right\rfloor$, is minimized. This happens if $u=H$, then the prover's computation is dominated by $\Theta(\log H)$ scalar multiplications and exponentiations. Moreover, in this case the CRS length 
$n^{1+o(1)}$ is constant. Finally, we might want the summatory length of the CRS and the communication to be minimal, that is, $n^{1+o(1)}+\Theta\left(n_{v}\right)$. Considering $n \approx \log _{u} H$ and $n_{v} \approx \log _{2} u$, we get that the sum is $(\log H / \log u)^{1+o(1)}+\Theta(\log u)$. One can approximately minimize the latter by choosing $u=e^{\sqrt{\ln H}}$. Then the summatory length is $\log ^{1 / 2+o(1)} H$. (In this case, it would make sense to change the role of groups $\mathbb{G}_{1}$ and $\mathbb{G}_{2}$ to get better efficiency.) The efficiency of the new range proof in all three cases is given in Tbl. 1 .

Acknowledgments. The authors were supported by Estonian Science Foundation, grant \#9303, and European Union through the European Regional Development Fund. The first author was also supported by European Social Fund's Doctoral Studies and Internationalization Programme DoRa.

\section{References}

1. Barreto, P.S.L.M., Naehrig, M.: Pairing-Friendly Elliptic Curves of Prime Order. In: Preneel, B., Tavares, S. (eds.) SAC 2005. LNCS, vol. 3897, pp. 319-331. Springer, Heidelberg (2006)

2. Boneh, D., Boyen, X., Shacham, H.: Short Group Signatures. In: Franklin, M. (ed.) CRYPTO 2004. LNCS, vol. 3152, pp. 41-55. Springer, Heidelberg (2004)

3. Camenisch, J., Chaabouni, R., Shelat, A.: Efficient Protocols for Set Membership and Range Proofs. In: Pieprzyk, J. (ed.) ASIACRYPT 2008. LNCS, vol. 5350, pp. 234-252. Springer, Heidelberg (2008)

4. Chaabouni, R., Lipmaa, H., Shelat, A.: Additive Combinatorics and Discrete Logarithm Based Range Protocols. In: Steinfeld, R., Hawkes, P. (eds.) ACISP 2010. LNCS, vol. 6168, pp. 336-351. Springer, Heidelberg (2010)

5. Di Crescenzo, G., Herranz, J., Sáez, G.: Reducing Server Trust in Private Proxy Auctions. In: Katsikas, S.K., López, J., Pernul, G. (eds.) TrustBus 2004. LNCS, vol. 3184, pp. 80-89. Springer, Heidelberg (2004)

6. Elkin, M.: An Improved Construction of Progression-Free Sets. Israeli Journal of Mathematics 184, 93-128 (2011)

7. Groth, J.: Honest Verifier Zero-Knowledge Arguments Applied. PhD thesis, University of Århus, Denmark (October 2004)

8. Groth, J.: Short Pairing-Based Non-interactive Zero-Knowledge Arguments. In: Abe, M. (ed.) ASIACRYPT 2010. LNCS, vol. 6477, pp. 321-340. Springer, Heidelberg (2010)

9. Groth, J.: Efficient Zero-Knowledge Arguments from Two-Tiered Homomorphic Commitments. In: Lee, D.H., Wang, X. (eds.) ASIACRYPT 2011. LNCS, vol. 7073, pp. 431-448. Springer, Heidelberg (2011)

10. Groth, J., Sahai, A.: Efficient Non-Interactive Proof Systems for Bilinear Groups. Technical Report 2007/155, International Association for Cryptologic Research (April 27, 2007), http://eprint.iacr.org/2007/155 (version 20100222:192509) (retrieved in December 2011)

11. Groth, J., Sahai, A.: Efficient Non-interactive Proof Systems for Bilinear Groups. In: Smart, N. (ed.) EUROCRYPT 2008. LNCS, vol. 4965, pp. 415-432. Springer, Heidelberg (2008)

12. Hess, F., Smart, N.P., Vercauteren, F.: The Eta Pairing Revisited. IEEE Transactions on Information Theory 52(10), 4595-4602 (2006) 
13. Lipmaa, H.: On Diophantine Complexity and Statistical Zero-Knowledge Arguments. In: Laih, C.-S. (ed.) ASIACRYPT 2003. LNCS, vol. 2894, pp. 398-415. Springer, Heidelberg (2003)

14. Lipmaa, H.: Progression-Free Sets and Sublinear Pairing-Based Non-Interactive Zero-Knowledge Arguments. Technical Report 2011/009, International Association for Cryptologic Research (January 5, 2011), http://eprint.iacr.org/2011/009

15. Lipmaa, H.: Progression-Free Sets and Sublinear Pairing-Based Non-Interactive Zero-Knowledge Arguments. In: Cramer, R. (ed.) TCC 2012. LNCS, vol. 7194, pp. 169-189. Springer, Heidelberg (2012)

16. Lipmaa, H., Asokan, N., Niemi, V.: Secure Vickrey Auctions without Threshold Trust. In: Blaze, M. (ed.) FC 2002. LNCS, vol. 2357, pp. 87-101. Springer, Heidelberg (2003)

17. Pereira Geovandro, C.C.F., Simplício Jr., M.A., Naehrig, M., Barreto, P.S.L.M.: A Family of Implementation-Friendly BN Elliptic Curves. Journal of Systems and Software 84(8), 1319-1326 (2011)

18. Rial, A., Kohlweiss, M., Preneel, B.: Universally Composable Adaptive Priced Oblivious Transfer. In: Shacham, H., Waters, B. (eds.) Pairing 2009. LNCS, vol. 5671, pp. 231-247. Springer, Heidelberg (2009)

19. Sanders, T.: On Roth's Theorem on Progressions. Annals of Mathematics 174(1), 619-636 (2011)

20. Tao, T., Vu, V.: Additive Combinatorics. Cambridge Studies in Advanced Mathematics. Cambridge University Press (2006)

21. Yuen, T.H., Huang, Q., Mu, Y., Susilo, W., Wong, D.S., Yang, G.: Efficient Noninteractive Range Proof. In: Ngo, H.Q. (ed.) COCOON 2009. LNCS, vol. 5609, pp. 138-147. Springer, Heidelberg (2009)

\section{A Proof of Thm. 1}

Proof. PeRfeCt COMPleteness: correctness verifications are straightforward. Clearly,

$$
\begin{aligned}
\hat{e}\left(f, C_{f}\right) & =\hat{e}\left(f, g_{2}^{R_{f}} g_{2, \lambda_{1}}^{r_{f}}\right)=\hat{e}\left(f, g_{2}^{R_{f}}\right) \cdot \hat{e}\left(f, g_{2, \lambda_{1}}^{r_{f}}\right)=\hat{e}\left(f^{R_{f}}, g_{2}\right) \cdot \hat{e}\left(f^{r_{f}}, g_{2, \lambda_{1}}\right) \\
& =\hat{e}\left(\psi_{f}, g_{2}\right) \cdot \hat{e}\left(A_{f}, g_{2, \lambda_{1}}\right) .
\end{aligned}
$$

Analogously, $\hat{e}\left(h, C_{h}\right)=\hat{e}\left(\psi_{h}, g_{2}\right) \cdot \hat{e}\left(A_{h}, g_{2, \lambda_{1}}\right)$. Finally, $\hat{e}\left(A_{c} \psi_{g}^{-1}, g_{2}\right)$. $\hat{e}\left(g_{1}, C_{f} C_{h}\right)=\hat{e}\left(g_{1}^{r} g_{1, \lambda_{1}}^{a} \cdot g_{1}^{-r-R_{f}-R_{h}}, g_{2}\right) \cdot \hat{e}\left(g_{1}, g_{2}^{R_{f}+R_{h}}\right) \cdot \hat{e}\left(g_{1}, g_{2, \lambda_{1}}^{r_{f}+r_{h}}\right)=\hat{e}\left(g_{1, \lambda_{1}}^{a}\right.$. $\left.g_{1}^{-R_{f}-R_{h}}, g_{2}\right) \cdot \hat{e}\left(g_{1}^{R_{f}+R_{h}}, g_{2}\right) \cdot \hat{e}\left(g_{1}^{r_{f}+r_{h}}, g_{2, \lambda_{1}}\right)=\hat{e}\left(g_{1}^{a}, g_{2, \lambda_{1}}\right) \cdot \hat{e}\left(g_{1}^{r_{f}+r_{h}}, g_{2, \lambda_{1}}\right)=$ $\hat{e}\left(g_{1}^{r_{f}+r_{h}+a}, g_{2, \lambda_{1}}\right)$.

Computational Soundness: By the $\left\{1-X^{\lambda_{1}}\right\}$-PKE assumption in $\mathbb{G}_{1}$ and $\mathbb{G}_{2}$, one can open the next values: $\left(A_{c}, \bar{A}_{c}\right)=\left(g_{1}^{r} g_{1, \lambda_{1}}^{a}, \bar{g}_{1}^{r} \bar{g}_{1, \lambda_{1}}^{a}\right),\left(A_{g} / A_{c}, \AA_{g / c}\right)=$ $\left(\left(g_{1} g_{1, \lambda_{1}}^{-1}\right)^{a^{\prime}}, \stackrel{\circ}{g}_{1, g / c}^{a^{\prime}}\right),\left(A_{g}, \stackrel{\circ}{A}_{g}\right)=\left(g_{1}^{a^{\prime \prime}}, \stackrel{\circ}{g}_{1}^{a^{\prime \prime}}\right),\left(A_{f}, \stackrel{\circ}{A}_{f}\right)=\left(f^{r_{f}}, \stackrel{\circ}{f}^{r_{f}}\right),\left(A_{h}, \stackrel{\circ}{A}_{h}\right)=$ $\left(h^{r_{h}}, \stackrel{\circ}{h}^{r_{h}}\right),\left(C_{f}, \bar{C}_{f}\right)=\left(g_{2}^{R_{f}} g_{2, \lambda_{1}}^{r_{f}^{\prime}}, \bar{g}_{2}^{R_{f}} \bar{g}_{2, \lambda_{1}}^{r_{f}^{\prime}}\right),\left(C_{h}, \bar{C}_{h}\right)=\left(g_{2}^{R_{h}} g_{2, \lambda_{1}}^{r_{h}^{\prime}}, \bar{g}_{2}^{R_{h}} \bar{g}_{2, \lambda_{1}}^{r_{h}^{\prime}}\right)$, $\left(\psi_{g}, \stackrel{\circ}{\psi}_{g}\right)=\left(g_{1}^{r_{a}^{\prime \prime}}, \stackrel{\stackrel{r}{g}^{\prime \prime}}{r_{a}^{\prime \prime}}\right),\left(\psi_{f}, \stackrel{\circ}{\psi}_{f}\right)=\left(g_{1}^{r_{f}^{\prime \prime}}, \stackrel{\stackrel{r}{g}^{\prime \prime \prime}}{1, f}\right)$, and $\left(\psi_{h}, \stackrel{\circ}{\psi}_{h}\right)=\left(g_{1}^{r_{h}^{\prime \prime}}, \stackrel{\stackrel{g^{\prime}}{r_{h}^{\prime \prime}}}{1, h}\right)$. 
Since $A_{c}=g_{1}^{r} g_{1, \lambda_{1}}^{a}, A_{g}=g_{1}^{a^{\prime \prime}}$ and $A_{g} / A_{c}=\left(g_{1} g_{1, \lambda_{1}}^{-1}\right)^{a^{\prime}}$, we have that $g_{1}^{a^{\prime \prime}}=$ $g_{1}^{r+a^{\prime}} g_{1, \lambda_{1}}^{a-a^{\prime}}$. Thus, if $a \neq a^{\prime}$, one can compute $x^{\lambda_{1}} \leftarrow\left(a^{\prime \prime}-r-a^{\prime}\right) /\left(a-a^{\prime}\right)$, and from this compute $x$ and thus break the $\left\{1-X^{\lambda_{1}}\right\}$-PSDL assumption. (To verify whether $x$ is the correct root, one can check whether $g_{1}^{x^{\lambda_{1}}}=g_{1, \lambda_{1}}$.) Thus $a=a^{\prime}$, and thus also $a^{\prime \prime}=r+a$ and $A_{g}=g_{1}^{r+a}$.

Due to $C_{f}=g_{2}^{R_{f}} g_{2, \lambda_{1}}^{r_{f}^{\prime}}, \psi_{f}=g_{1}^{r_{f}^{\prime \prime}}, A_{f}=f^{r_{f}}$ and $\hat{e}\left(f, C_{f}\right)=\hat{e}\left(\psi_{f}, g_{2}\right)$. $\hat{e}\left(A_{f}, g_{2, \lambda_{1}}\right)$, we have $\hat{e}\left(f, g_{2}^{R_{f}} g_{2, \lambda_{1}}^{r_{f}^{\prime}}\right)=\hat{e}\left(g_{1}^{r_{f}^{\prime \prime}}, g_{2}\right) \hat{e}\left(f^{r_{f}}, g_{2}^{x^{\lambda_{1}}}\right)$ for unknown $x$. Taking the discrete logarithm of the both sides of the last equation, we get that $R_{f} / \mathrm{sk}_{1}+r_{f}^{\prime} x^{\lambda_{1}} / \mathrm{sk}_{1}=r_{f}^{\prime \prime}+r_{f} x^{\lambda_{1}} / \mathrm{sk}_{1}$, or $\left(r_{f}-r_{f}^{\prime}\right) x^{\lambda_{1}}=R_{f}-r_{f}^{\prime \prime} \cdot \mathrm{sk}_{1}$. Thus, if $r_{f} \neq r_{f}^{\prime}$, then we can compute $x^{\lambda_{1}}$, and find from this $x$, and thus break the $\left\{1-X^{\lambda_{1}}\right\}$-PSDL assumption. Thus, $r_{f}=r_{f}^{\prime}$ and therefore also $C_{f}=g_{2}^{R_{f}} g_{2, \lambda_{1}}^{r_{f}}$. Moreover, $\psi_{f}=g_{1}^{r_{f}^{\prime \prime}}=f^{R_{f}}$.

Analogously, we get that $r_{h}=r_{h}^{\prime}$ and therefore $C_{h}=g_{1}^{R_{h}} g_{1, \lambda_{1}}^{r_{h}}$ and $\psi_{h}=h^{R_{h}}$. Due to $C_{f}=g_{2}^{R_{f}} g_{2, \lambda_{1}}^{r_{f}}, C_{h}=g_{1}^{R_{h}} g_{1, \lambda_{1}}^{r_{h}}, \psi_{g}=g_{1}^{r_{a}^{\prime \prime}}, A_{c}=g_{1}^{r} g_{1, \lambda_{1}}^{a}, A_{g}=g_{1}^{r+a}$ and $\hat{e}\left(g_{1}, C_{f} C_{h}\right)=\hat{e}\left(\psi_{g} A_{c}^{-1}, g_{2}\right) \cdot \hat{e}\left(A_{g}, g_{2, \lambda_{1}}\right)$, we have $\hat{e}\left(g_{1}, g_{2}^{r+R_{f}+R_{h}+\left(r_{f}+r_{h}\right) x^{\lambda_{1}}}\right)=$ $\hat{e}\left(g_{1}^{r_{a}^{\prime \prime}} g_{1}^{-r} g_{1, \lambda_{1}}^{-a}, g_{2}\right) \cdot \hat{e}\left(g_{1}^{r+a}, g_{2, \lambda_{1}}\right)=\hat{e}\left(g_{1}^{r_{a}^{\prime \prime}-r+r x^{\lambda_{1}}}, g_{2}\right)$ for unknown $x$. Taking the discrete logarithm of both sides of the last equation, we get $r+R_{f}+R_{h}+\left(r_{f}+\right.$ $\left.r_{h}\right) x^{\lambda_{1}}=r_{a}^{\prime \prime}-r+r x^{\lambda_{1}}$. Again, if $r_{f}+r_{h} \neq r$, then one can compute $x^{\lambda_{1}}$ and thus also $x$. Thus, $r=r_{f}+r_{h}$, and thus also $r_{a}^{\prime \prime}=r+R_{f}+R_{h}$. This means that $A_{c}=g_{1}^{r_{f}+r_{h}} g_{1, \lambda_{1}}^{a}$ and $\left(A_{g}, A_{f}, A_{h}\right)=\left(g_{1}^{r_{f}+r_{h}+a}, f^{r_{f}}, h^{r_{h}}\right)$.

COMPutational ZERO-KNOWLEDGE: we construct the next simulator $\left(\mathcal{S}_{1}, \mathcal{S}_{2}\right) . \mathcal{S}_{1}$ creates a CRS according to the protocol together with a trapdoor $\mathrm{td}=\left(\alpha_{g}, \alpha_{f}, \alpha_{h}, \bar{\alpha}, \alpha_{g, c}, x\right)$. On input td, $\mathcal{S}_{2}$ creates $z_{f}, z_{h} \leftarrow \mathbb{Z}_{p}$. He then sets $C_{f} \leftarrow g_{2}^{z_{f}}, \psi_{f} \leftarrow f^{z_{f}} / A_{f}^{x^{\lambda_{1}}}, C_{h} \leftarrow g_{2}^{z_{h}}, \psi_{h} \leftarrow h^{z_{h}} / A_{h}^{x^{\lambda_{1}}}$, and $\psi_{g} \leftarrow g_{1}^{z_{f}+z_{h}} / A_{g}^{x^{\lambda_{1}}}$. He creates the knowledge elements $\left(\AA_{g}, \AA_{f}, \AA_{h}, \stackrel{\AA}{A}_{c}, \stackrel{\circ}{\psi}_{g}, \bar{C}_{f}, \stackrel{\circ}{\psi}_{f}, \bar{C}_{h}, \stackrel{\circ}{\psi}_{h}, \stackrel{\AA}{A}_{g / c}\right)$ by using the trapdoor. For example, $\AA_{g / c} \leftarrow\left(A_{g} / A_{c}\right)^{\alpha_{g / c}}$. One can now check that the verification succeeds. For example, $\hat{e}\left(\psi_{f}, g_{2}\right) \hat{e}\left(A_{f}, g_{2, \lambda_{1}}\right)=\hat{e}\left(f^{z_{f}} / A_{f}^{x^{\lambda_{1}}}, g_{2}\right)$. $\hat{e}\left(A_{f}, g_{2, \lambda_{1}}\right)=\hat{e}\left(f^{z_{f}}, g_{2}\right) / \hat{e}\left(A_{f}^{x^{\lambda_{1}}}, g_{2}\right) \hat{e}\left(A_{f}, g_{2, \lambda_{1}}\right)=\hat{e}\left(f^{z_{f}}, g_{2}\right)=\hat{e}\left(f, C_{f}\right)$, and finally, $\hat{e}\left(A_{c} \psi_{g}^{-1}, g_{2}\right) \cdot \hat{e}\left(g_{1}, C_{f} C_{h}\right)=\hat{e}\left(g_{1}^{-z_{f}-z_{h}} A_{g}^{x^{\lambda_{1}}} A_{c}, g_{2}\right) \cdot \hat{e}\left(g_{1}, g_{2}^{z_{f}+z_{h}}\right)=$ $\hat{e}\left(A_{g}, g_{2, \lambda_{1}}\right)$. If the DLIN assumption is true, then $\left(A_{g}, A_{f}, A_{h}\right)$ is indistinguishable from an encryption of $0 \in[0, H]$, and thus the whole argument is computationally zero-knowledge. 